\title{
MECHANICS/HEAT-TRANSFER RELATION FOR PARTICULATE MATERIALS
}

Final Report

By

Charles S. Campbell

David G. Wang

Khurram Rahman

November 1991

Work Performed Under Contract No. FG22-88PC88913

For

U.S. Department of Energy

Pittsburgh Energy Technology Center

Pittsburgh, Pennsylvania

By

University of Southern Callfornia

Los Angeles, Callfornla 


\section{DISCLAIMER}

This report was prepared as an account of work sponsored by an agency of the United States Government. Neither the United States Government nor any agency thereof, nor any of their employees, makes any warranty, express or implied, or assumes any legal liability or responsibility for the accuracy, completeness, or usefulness of any information, apparatus, product, or process disclosed, or represents that its use would not infringe privately owned rights. Reference herein to any specific commercial product, process, or service by trade name, trademark, manufacturer, or otherwise does not necessarily constitute or imply its endorsement, recommendation, or favoring by the United States Government or any agency thereof. The views and opinions of authors ex. pressed herein do not necessarily state or reflect those of the United States Government or any agency thereof.

This report has been reproduced directly from the best available copy.

Available to DOE and DOE contractors from the Office of Scientific and Techuical Information, P.O. Box 62, Oak Ridge, TN 37831; prices available from (615)576-8401, FTS 626-8401.

Available to the public from the National Technical Information Service, U.S. Department of Cominerce, 5285 Port Royal Rd., Springfield, VA 22161. 
$\mathrm{DOE} / \mathrm{PC} / 88913--\mathrm{T} 10$

DE92 007934

\section{MECHANICS/HEAT-TRANSFER RELATION FOR PARTICULATE MATERIALS}

Grant \#DE-FG22-88PC88913

Final REPORT

November, 1991

Report DOEHT.FN

Charles S. Campbell

David G. Wang

$\&$

Khurram Rahman

Department of Mechanical Engineering

University of Southern California

Los Angeles, Ca. 90089-1453 


\section{SUMMARY}

The original goal of this study was to try and understand the relationship between the thermal and mechanical properties of particulate flows.

Two situations were examined. The first is a study of the effects of simple shear flows, as a embryonic flow type on the apparent thermal conductivity and apparent viscosity of a dry granular flow. This program has demonstrated that both quantities increase linearly with the imposed velocity gradient, implying that both are driven by the square root of the granular temperature (just as the viscosity and thermal conductivity of a billiard ball gas varies as the square root of the thermodynamic temperature). In addition, the studies the differences in the mechanism of internal transport of the two quantities. They indicate that both heat and momentum may be transported in the streaming mode, i.e. by the random motions of the constituent particles, but, as heat transfer is a relatively slow process, only momentum is exchanged in interparticle collisions.

The second study involved fluidized beds. The original idea was to try and relate the heat transfer behavior of a fluidized bed to the "particle pressure", the forces by only the particle phase of the twophase mixture. This part of the study was really two steps in itself. While terms involving the particle pressure and other interparticle forces have long appeared in multiphase now models, these terms have never been measured. Consequently, there is great value in simply measuring the particle pressures. We performed this study in a gas nuidized bed and determined that the principle generation of particle pressure came from the motion of bubbles and that the particle pressure could be related to the bubble size. The second phase of the study was to relate the particle pressure to the heat transfer. This. unfortunately proved unsuccessful. 


\section{TABLE OF CONTENTS}

1.0 Introduction 1

2.0 Reynolds' Analogy for Particle Flows: The Relationship between the 3 Apparent Thermal Conductivity and Viscosity for a Sheared Granular Material

2.1 Experimental Apparatus 6

2.2 Experimental Procedure 11

2.3 Calculation of the Apparent Thermal Conductivity 12

2.4 Results and Discussion 14

2.5 Conclusions 23

3.0 Particle Pressures in Gas Fluidized Beds 25

$\begin{array}{ll}3.1 & \text { The Particle Pressure Transducer }\end{array}$

3.2 Testing of the Particle Pressure Transducer 28

3.3 Fluidized Bed 33

3.4 Particle: Pressures in Gas-Fluidized Beds ' 33

3.5 The New Particle Pressure Transducer 44

3.6 Particle Pressure in Gas-Fluidized Beds Undergoing Particulate Fluidization $\quad 46$

3.7 The Relationship between the Particle Pressure and Heat Transfer 50

$\begin{array}{lll}3.8 & \text { Conclusions } & 52\end{array}$

4.0 Appendix - Experimental Innovations 54

4.1 Heater Control Circuit 54

4.2 Thermocouple Connections through Slip Rings 55

$\begin{array}{ll}\text { References } & 58\end{array}$ 


\section{CIIAPTER 2: REYNOLDS' ANALOGY FOR PARTICLE FLOWS: TIE RELATIONSIIP BETWEEN TIE APPARENT THERMAL CONDUCTIVITY AND VISCOSITY FOR A SIEARED GRANULAR MATERIAL}

"Reynolds analogy" for fluids is a term that is commonly used to describe relationships between their mechanical behavior - e.g. the shear force exerted on a surface - and their thermal behavior - e.g. the heat transfer from that surface. Such relationships exist because similar mechanisms govern the microscopic transport of both properties internal to the material. On a molecular level, the mechanism that drives the transport of both quantities is the random motions of the molecules which is characterized by the thermodynamic temperature. The macroscopic manifestations of this internal transport are the viscosity and thermal conductivity of the material. Similarly, both heat and momentum are carried by the eddies that form within a turbulent fluid, leading to the so-called "eddy" viscosity and thermal conductivity. (It was in this context that Reynolds (1874) first proposed the analogy.) Given such strong relationships between the internal, microscopic transport of both quantities, it should not be surprising to find relationships between the resulting macroscopic surface skin friction and heat transfer.

A rapidly sheared granular material has much in common with both molecular and turbulent transport. There, collisions, induced by the shear now, induce random motions of the particles that strongly invoke the image of the thermalized motion of molecules in the kinetic theory of gases. The image is so strong that the energy associated with the random movements is characterized as a "granular temperature." This has given rise to a school of thought known as rapid granular nlow theory, (see the review by Campbell (1990)), which tries to understand the mechanics of rapidly sheared granular materials based largely on ideas derived from kinetic theory. But unlike a real thermodynamic material, the collisions between macroscopic solid particles are inelastic. As a result, the granular temperature cannot be self sustaining, but must be continually supplied with energy from the motion of the bulk material. Thus, like the eddies in a turbulent fluid, the granular temperature is a property of the granular flow and not a property of the granular material. And, like both the thermodynamic temperature and nuid turbulence, the random particle motions reflected in the granular temperature can induce the transport of both heat and momentum leading one to expect that some relationship must exist between the macroscopic transport properties of both.

The literature contains several precedents for such a conclusion. For example, Patton et al. (1986) measured the friction and the heat transfer between a dry granular material and a flat plate embedded in the bottom of an inclined chute flow. They found that, as for a nuid now, a simple relationship existed between the friction coefficient and Stanton number even though, individually, their behavior is 
quite complex. A much closer relationship exists for suspensions. Leal (1973) analyzed the apparent conductivity of a diiute suspension of neutrally buoyant spherical drops in the limit of low particle Reynolds and Peclet numbers in a simple shear now. The analysis assumed that the imposed velocity and temperature gradients both point in the same direction. In such a case, as the bulk shear motion is perpendicular to the temperature gradient, it can have no direct effect on the heat or momentum transport, so that any change in the thermal conductivity or viscosity must come from the the perturbation to the nuid motion induced by the presence of the particle, or, perhaps, from the shear induced rotation of the particle itself. Leal calculated that the shear flow enhanced the bulk thermal conductivity proportional to the $\frac{3}{2}$ power of the shear rate, a conclusion which was confirmed experimentally by Chung \& Leal (1982). A similar analysis for the apparent viscosity by Lin, Peery \& Schowalter (1970) showed the same $\frac{3}{2}$ power law dependence of the apparent viscosity on the shear rate, again, indicating a close connection between the internal mechanisms of heat and momentum transport.

But for macroscopic materials, it is possible that the mechanisms that lead to heat and momentum transport may become uncoupled. This is evident in the work of Nir \& Acrivos (1976) who studied the effective thermal conductivity in the limit of low Reynolds number but large Peclet number (indicating a large Prandtl number fluid). In such a case, the mechanisms of momentum transport are unchanged from the case studied by Lin, Peery \& Schowalter (1970), but the heat transport is confined to a thin boundary layer about a closed streamline pattern that forms around the particle. In such a case, the conductivity is only weakly dependent on shear rate and varies proportional to $\mathrm{Pe}^{1 / 11}$. Here, the heat and momentum transport become uncoupled as the material properties dictate that the major players in the heat transport make only a minor contribution to the momentum transport; all of the heat transport occurs within the interstitial fluid and the particle only has an influence because of the perturbation it produces within the pattern of nuid now. This has no direct analog for dry granular nows as, there, the thermal properties of the nuid (conductivity, heat capacity, etc.) are insignificant compared to that of the solid, and the motion of the nuid induces an insignificant amount of heat transport compared to that induced by the motion of particles. However, there still are mechanisms that contribute either to the transport of heat or the transport of momentum without affecting the transport of the other quantity. One example, on the heat transfer side, is particle rotation. Campbell (1989) showed that, in a dry granular flow, the bulk shear motion induces a particle rotation rate of the order of one-half the shear rate - much as is observed for particles in a fluid shear flow. By itself, the rotation of the particle does not transport linear momentum and thus cannot contribute directly to the stress. However, it does induce a convective transport of heat as a rotating particle within a temperature gradient will absorb heat on the hot side and release it on the cold side. The case of a particle rotating in a stationary medium has been studied by Wang, Sadhal, \& Campbell (1989), who 
showed that the major effect of the particle rotation is to intrease the apparent conductivity of the particle relative to that of the fluid; thus, the maximum thermal conductivity of an assembly of rotating particles can be no larger than that of an assembly of stationary particles whose conductivity is infinite compared to that of the medium within which they are embedded. Thus, for a dry granular flow, as the conductivity of the solid phase is already much larger than that of the interstitial fluid, the amount of additional heat transported due to the particle rotation is insignificant.

The current study is carried out to experimentally measure the apparent thermal conductivity and the apparent viscosity of a dense dry particulate miterial as it undergoes shear in an annular shear cell. Here the particle concentration, particle size and shear rate are much larger- indicating large Re and $\mathrm{Pe}$ - relative to the suspension studies referred to above; as such, while there may still be a relationship between the thermal conductivity asd viscosity, one should not be surprised that the two studies will show significantly different results. In fact, such a difference is already apparent in extensive studies of the mechanical behavior of such systems, both by direct experimentation (Bagnold (1954), Hanes \& Inman (1985), Savage \& Sayed (1983)) and by computer simulation, (Campbell (1982, 1989), Campbell \& Brennen (1985), Campbell \& Gong (1986) and Walton \& Braun (1986ab), (there have been no previous measurements of the thermal conductivity). The pioneering work on the constitutive behavior of momentum transfer for granular materials was Bagnold's (1954) study of the shear flows of neutrally buoyant wax spherical particles suspended in a glycerine-water-alcohol solution. He showed that, at low shear rates, the shearing mixture behaves like a Newtonian fluid with a corrected viscosity. At large shear rates, however, the presence of the particles plays a more important role; there, the composite ceases to behave like a Newtonian nuid and the stresses, $\tau$, vary as the square of the bulk velocity gradient according to the rule:

$$
\tau=\rho_{\mathrm{p}} \mathrm{D}_{\mathrm{p}}^{2} \mathrm{f}(\nu) \gamma^{2}
$$

where $\rho_{p}$ is the density of the solid particles, $D_{p}$ the particle diameter, $\nu$ the solid fraction defined as the fraction of the unit volume occupied by the solid phase, $\gamma$ is the bulk shear rate and $f(\nu)$ a function of the solid fraction, $\nu$ (a dimensionless density equal to the fraction of volume occupied by solid material). The same conclusion may be derived by dimensional analysis. The quadratic dependence of the stresses on the shear rate described by Equation $(2.0 .1)$ has been confirmed by all of the experiments and computer simulation studies referred to above. The implication of this result is that the apparent viscosity - i.e. the shear stress divided by the shear rate - varies directly proportional to the shear rate. 


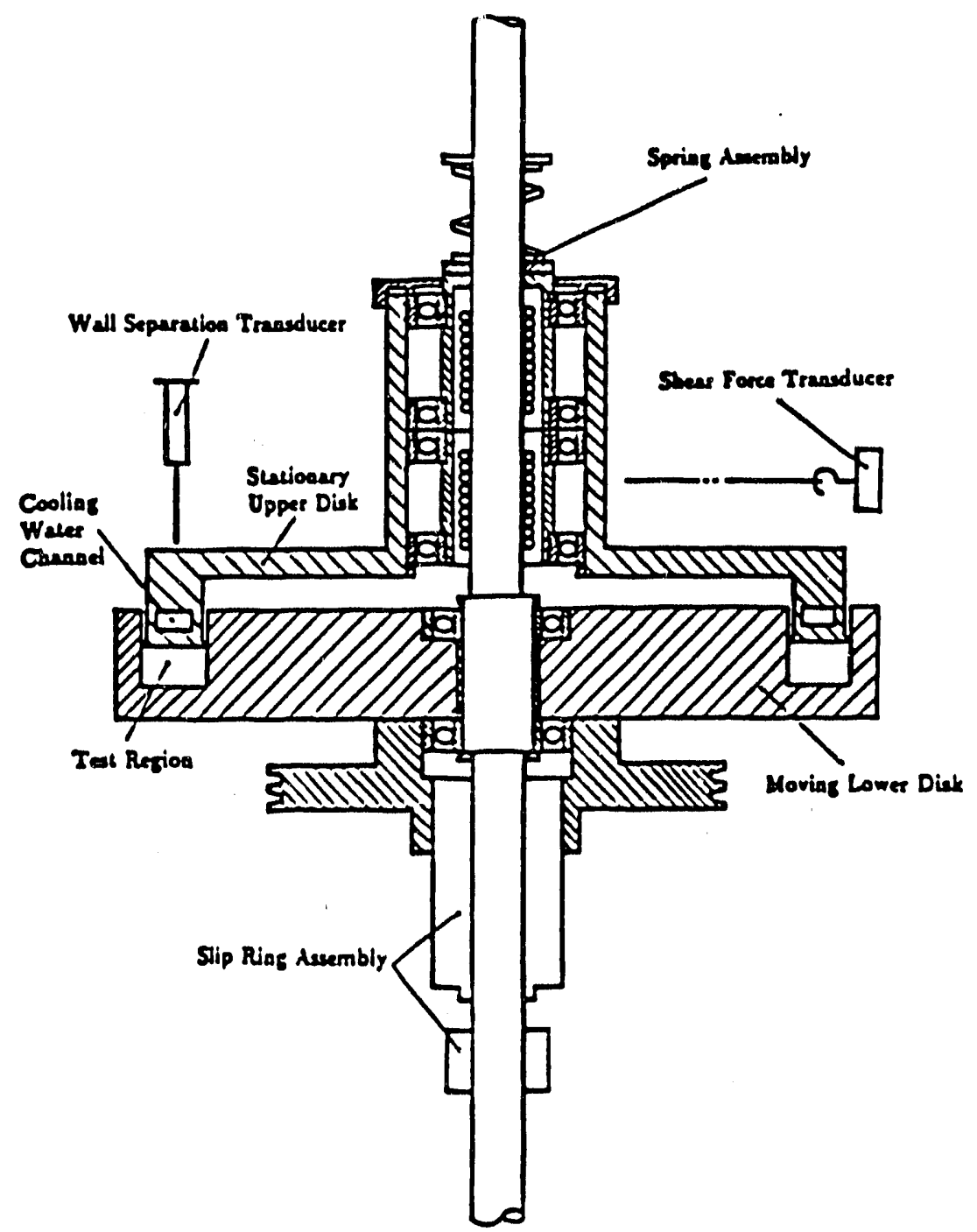

Figure 2.1.1: Schematic of the shear cell.

\subsection{EXPERIMENTAL APPARATUS}

The apparatus used to measure the apparent conductivity is shown in Figure 2.1.1. It is an annular shear cell similar to those used by Hanes \& Inman (1985) and Savage \& Sayed (1983) for their studics of the mechanical behavior of sheared granular materials - although, here, the basic design has been modified so that it may simultaneously measure the apparent thermal conductivity. It consists of a moving lower disk and a stationary upper disk coaxially mounted on a central shaft. The low $r$ disk is made of nylon and is $610^{\mathrm{mm}}\left(24^{\text {in }}\right)$ in diameter and $76^{\mathrm{mm}}\left(3^{\text {in }}\right)$ thick, and is mounled on a radial bearing assembly along the shaft which allows the disk to sotate freely. An annular trough of $584 \mathrm{~mm}$ $\left(23^{\text {in }}\right)$ in outside diameter, $483^{\mathrm{mm}}\left(19^{\text {in }}\right)$ in inside diameter and $51^{\mathrm{mm}}\left(2^{\text {in }}\right)$ deep is cut into the disk to hold the heater assembly, thermocouples and test materials. The disk is belt driven by a 5 HP AC 
motor whose speed is controlled by a variable speed inverter. All of the cell's construction materials must be extremely temperature tolerant as all of the mechanical energy (up to the full $5 \mathrm{HP}$ of the drive motor) is eventually dissipated away to heat and the internal temperatures may exceed $100^{\circ} \mathrm{C}$. Yet the use of metals, (an obvious choice because of their resistance to abrasion and temperature) is inad. isable for the construction of the lower disk as their large thermal conductivity, relative to the test material, might bias the measurement. After several attempts, the lower disk was eventually constructed of Nylon as it is able to withstand larger temperatures than many other commonly available plastics, while possessing a thermal conductivity an order of magnitude smaller than those of metals.

The upper stationary disk is made of aluminum and machined to close fit into the trough of the lower disk without directly contacting the trough walls. A cooling water channel is recessed right behind the surface to keep the top wall temperature constant during the heat transfer experiments. The cooling water is supplied by a Fisher Scientific constant temperature bath. The disk is mounted on a radial-linear bearing assembly to allow free rotational and axial movement. However, rotation of the disk is prevented by a cable connected to a load cell which measures the shear force exerted on the top surface by the material. The axial movement of the top disk is restrained by a spring assembly which provides a normal force to the sheared mixture in the trough; the balance between the spring force and the shear induced dispersive stresses controls the height of the shear gap between upper and lower disks. The gap height is monitored by a LVDT gauge head. Both the top and bottom shearing surfaces are roughened by gluing on solid particles spaced several diameters apart so that good mechanical contact is insured between the test material and the shearing surfaces.

The heater assembly, shown in Figure 2.1.2, consists of six separate heaters which are mounted behind the roughened plate that covers the trough bottom. The primary heater is located at top center and is sandwiched by two guard heaters to prevent any heat nux through the metal plate in the direction parallel to the plate. A single ring-shaped bottom guard heater which spans the whole gap width is placed, behind a plastic spacer, at the back of the primary heater. Finally, two additional guard heaters are used to block the heat path through the sides of the plastic spacer and the roughened metal plate. To ensure that the outgoing energy from the primary heater goes nowhere but upward to the test material, the temperatures of all five guard heaters are kept the same as the temperature of the primary heater.

The measurements of temperature are made via T-type thermocosples whose positions are marked in Figure 2.1.2. Three thermocouples are placed on the primary heater in order to detect any temperature gradient along its width in radial direction. Three thermocouplis are placed along each of the shearing walls and four thermocouples along the vertical sidewalls of the channel to monitor the 


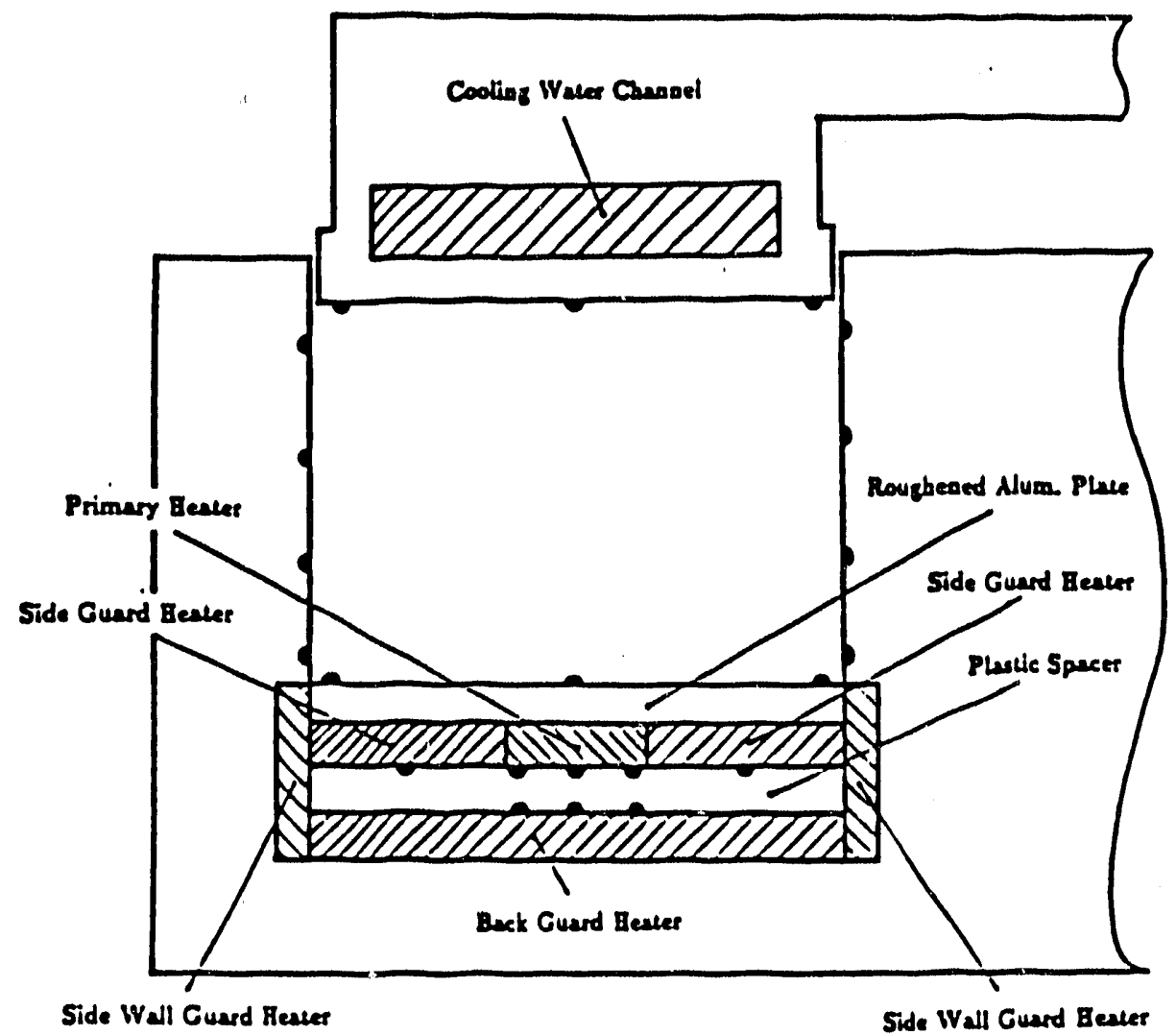

Figure 2.1.2: Schematic of the beater assembly and thermocouple placement

surface temperature distribution. A total of 19 thermocouples are mounted on the rotating disc, and along with twelve leads from the heater assembly, are connected to the stationary world through two slip rings assemblies. An additional three thermocouples are attached to the stationary upper surface. For the conductivity calculation, The temperature distribution along the two roughened boundaries is determined by three thermocouples mounted flush with the outer edges of the roughening particles.

One novel feature of this device is that the shear cell is mounted inside a rotatable cage to change the orientation of the shear zone with respect to gravity. In all previous experiments that employed similar apparatuses, including Ilanes \& Inman (1985) and Savage \& Sayed (1984), the orientation of the shear cell was fixed so that gravity worked in the same direction as the velocity gradient. Both investigators observed that, at low bulk densities, the material would gather near the trough bottom in a stagnant layer and only that portion of the matcrial above that layer would actually shear. Consequently, they were only able to operate the experiments in a fully shearing mode at very bigh solid concentrations. When the present apparatus is rotated so that the shaft is parallel to the ground, gravity works perpendicular to the velocity gradient, preventing the stagnant zone formation and 
permitting operation at solid concentrations as low as $5 \%$ by volume. The stress results at low concentrations have been shown by Campbell $(1986,1990)$ to agree with computer simulation results and are the only measurements at low concentrations that do so - indicating that this is a valid method of making measurements at such small concentration. In addition, the gravitational orientation varies about the circumference so that its net contribution averages to zero over a complete rotation. However, despite very successful operation at small concentrations, problems were encountered with the shear cell operated in this orientation at large concentrations. The problems appeared as vigorous oscillation of the top disk for $\nu>0.35$. Similar oscillations are obcerved, though, to a smaller degree, when the device was rotated into its more familiar orientation with the shaft vertical and may also be found described in the papers by Savage \& Sayed and Hanes \& Inman. It is speculated that at high densities, particles may percolate across the channel depth forming columns that span the gap width; as the top surface tries to move over such a column it may be temporary lifted, as on a pole vault, generating the observed oscillation. Apparently, with the shear cell rotated so that the shaft is horizontal, there is sufficient circumferential variation in the solid concentration to promote such events (at such large concentrations, only a small perturbation would be required). One would expect that these polevaults could transmit a force directly from the top to the bottom disk, which might bias the force measurement and it is unclear what the consequences would be for the conductivity measurement.. To aninimize this problem, the shear cell is reoriented with the shaft in the vertical direction, (so that gravity works parallel to the velocity gradient,) whenever the concentration is larger than $\nu=0.35$. Test were made to assure that the orientation change did not bias either the conductivity or viscosity measurements.

Even though changing the orientation eliminates the gravity induced formation of stagnant zones along the bottom of the channel, it still possible for centrifugal forces to force stagnant gions to form - especially in the outside lower corners of the trough. To ensure that shearing occurs across the whole shear depth of the trough, tests are undertaken to determine the depth of the shearing for various values of solid concentration and shear rate. To accomplish this, the outer wall of the trough was covered with a layer of chalk dust. After shearing, the particles are carefully unloaded and any stagnant regions could be seen as areas where the chalk dust was undisturbed. In this way, we found that the maximum gap spacing for which no stagnant zone could be observed was about 8.5 particle diameters. As a smaller shear gap presented the danger of significant influence of the bounding walls, this spacing was used throughout the experiments described here.

Although the use of this type of device has become quite ubiquitous for granular material testing, several problems with the measurements have made themselves apparent in recent years. The most obvious problems arise from the character of the roughed boundaries. This was first noted by Haries \& 
Inman (1985) who performed experiments with a shear cell that was identical, in nearly every respect, to that used by Savage \& Sayed (1984), except in the way the shearing boundaries were prepared. Savage \& Sayed roughened their boundaries by attaching coarse sandpaper, to the shearing surfaces, while Hanes \& Inman roughened theirs by gluing particles of the test material to the surfaces. Surprisingly, tests on nearly identical materials, produced results that differed by a factor of up to three. Later Craig et al. (1987) showed that the test material itself had less effect on the stress measurements than the way that the boundaries were prepared. The boundaries used in the currents study were prepared by individually gluing particles spaced several diameters apart; the computer simulation results of Campbell \& Gong (1.987), indicate that this should provide the roughest possible boundaries, assuring good mechanical contact between the driving boundaries and the test material. In addition, Löffelmann (1989), made measurements through clear side walls of his shear cell and fornd that the generated shear rate was not uniform. Consequently, it appears that measurements made in this type of sinear cell, like .nany rheological measurements, are device dependent and may not be quantifiably related to the properties of a material undergoing an ideal simple shearing motion.

The choice of possible test materials was extremely limiter, both by constraints on size and ability to withstand the large temperatures that are generated by mechanical dissipation within the material. The maximum sizes of the particles are somewhat restricted by the maximum gap width supportable by the the experimental apparatus. On the low end, tine size of the particles is limited by the clearances between the stationary upper surface and the walls of the annula: trough. Although originally machined to within a quarter millimeter the gap expands as the material heats until it exceeds a millimeter. This limits the size of test materials to be significantly larger than a millimeter. Furthermore, plastics are eliminated as candidates since the temperatures within the shear gap can reach $100^{\circ} \mathrm{C}$ or higher. The final choices were steel shot and various sizes of glassbeads whose physical properties are listed below in Table 2.1.1.

Table 2.1.1. Material Properties

\begin{tabular}{|c|c|c|c|}
\hline Material & $\begin{array}{c}\text { Diameter } \\
\mathrm{D}_{\mathrm{p}}(\mathrm{mm})\end{array}$ & $\begin{array}{c}\text { Density } \\
\rho_{\mathrm{p}}\left(\mathrm{kg} \mathrm{m}^{-3}\right)\end{array}$ & $\begin{array}{c}\text { Specific Heat } \\
\mathrm{c}_{\mathrm{p}}\left(\mathrm{J} \mathrm{kg}^{-1} \mathrm{~K}^{-1}\right)\end{array}$ \\
\hline Glassbeads & 1.9 & 2500 & 0.74 \\
\hline Steel shot & 2.2 & 7300 & 0.42 \\
\hline Glassbeads & 3.0 & 2500 & 0.74 \\
\hline Glassbeads & 3.75 & 2500 & 0.74 \\
\hline
\end{tabular}


Further details about the design, construction and use of this apparatus, may be found in Wang (1991).

\subsection{EXPERIMENTAL PROCEDURE}

A typical experiment starts with a known quantity of material which is loaded into the trough in the lower disk and preheated to the temperatures that are anticipated in the experiment. The upper disk is then lowered and the orientation of the shear cell is set appropriately according the criteria discussed in Section 2.1. The motor is started and the normal force spring is adjusted to keep constant wall separation. The shear stress, electrical power supplied to the primary heater, and the temperatures on all four bounding walls of the test region, are all monitored to determine when the system reaches an equilibrium state. This process usually takes one hour, but, in some cases, it could take up to three hours. Many problems arose from the need to perform the experiment over such long periods of time within the highly abrasive environment of a shzaring granular material.

To balance the heaters, the temperature of the primary heater is set and the power applied to the other heaters is varied until all the temperatures, measured by the appropriate thermocouples, match. Looking at Figure 2.1.2, one will notice that there are three thermocouples on the primary heater, thermocouples near the outer edges of the side guard heaters and no thermocouples on the wall guard heaters. The power input to the side guard heaters was set so that the readings from the three thermocouples along the primary heater were the same. This assured that there were no temperature gradients along the surface of the primary heater that would drive heat losses in that direction. The temperature of the wall guard heaters were then set to balance the temperature of the side guard heaters.

The greatest problem arises from the abrasive wear on the roughness elements attached to the shearing surfaces. The wear makes itself apparent as a decrease in the shear stress, indicating that there may be significant slip on the shearing surfaces. Clearly, the higher the shear rate imposed on the sample and the longer the test period, the greater the damage that occurs to the roughened surfaces. Throughout this study, the experiment was stopped and particles reglued whenever a significant change in the stress was detected. At solid fractions higher than 0.40 , the damage to the roughened surfaces occurred so rapidly that repair was required after each data point. As the repair requires that the experiment be shut down for a day for the glue to cure, it took a long time to perform the experiments described herein. 


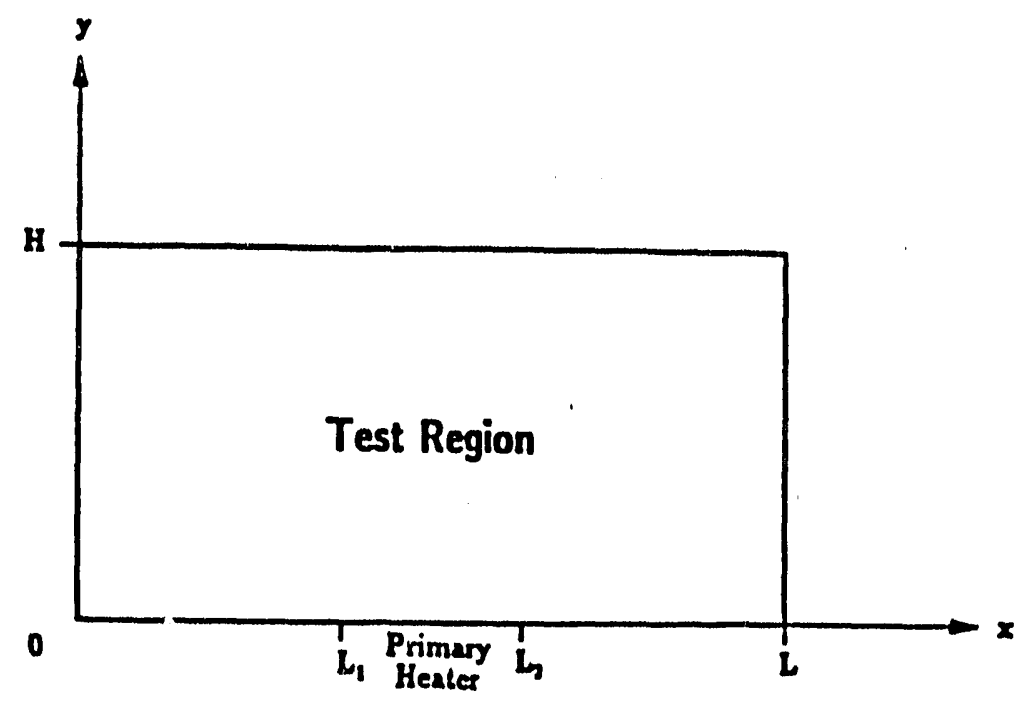

Figure 2.3.1: A cross-section of the annular test region upon which the conductivity calculation is performed.

\subsection{CALCULATION OF TUE APPARENT THERMAL CONDUCTIVITY}

The first step in finding the apparent thermal conductivity was to determine the temperature field which is calculated assuming homogeneous thermal properties for the material. Afterwards, the conductivity may be easily determined from the heat supplied by the primary heater and the temperature gradient at the wall. However, for the case studied here, there are two iactors that complicate such a calculation. The first problem arises because the annular region is nearly square in cross section and significant amounts of heat may be lost out the sidewalls, resulting in a two dimensional temperature field. In addition, the shearing process dissipates a great deal of heat and this must be taken into account in the calculation of the temperature field. Here, we make the assumption that the beat dissipation is uniformly distributed throughout the sample. (This is the most questionable assumption in the analysis. However, temperatures do not vary strongly along the top and bottom plates, indicating that the dissipation is, indeed, fairly uniform.) The total dissipation must equal the total work performed on the sample and is equal to $\mathrm{SU}$ where $\mathrm{S}$ is the total shear force measured by the shear force transducer and $U$ is the velocity of the lower plate. With these ideas in mind, the appropriate equation from which to find the temperatures is:

$$
k\left(\frac{\partial^{2} T}{\partial x^{2}}+\frac{\partial^{2} T}{\partial y^{2}}\right)+\frac{S U}{V}=0
$$


where $k$ is the apparent thermal conductivity of the shearing mixture, $T$ is the temperature, and $V$ the is total volume of the shearing region. This calculation is done on a cross-section of the test region that is illustrated in Figure 2.3.1. Here, $\mathrm{x}$ is the coordinate parallel to the roughened plates and varies between 0 and $L$ where $L$ is the width of the annulus; $y$ is the coordinate in the shear direction and varies between $\mathrm{O}$ and $\mathrm{H}$ where $\mathrm{H}$ is the height of the shear gap. Equation (2.3.1) is to be solved subject to the boundary conditions which are obtained by taking quadratic fits to the temperatures measured along the four walls.

$$
\begin{array}{ll}
T(x, 0)=c_{1} x^{2}+b_{1} x+a_{1} & \text { at } y=0 \\
T(0, y)=c_{2} y^{2}+b_{2} y+a_{2} & \text { at } x=0 \\
T(x, H)=c_{3} x^{2}+b_{3} x+a_{3} & \text { at } y=H \\
T(L, y)=c_{4} y^{2}+b_{4} y+a_{4} & \text { at } x=L
\end{array}
$$

with $a_{i}, b_{i}, c_{i}$ being the coefficients of thr quadratic fits to the temperature measurements on the boundaries. (On the top and bottom boundaries, the temperature measurement was made at the top of the roughness elements.) Now there are four thermocouples mounted on the sidewalls in the y-direction. Throughout these experiments, the gap, $H$, is varied to maintain a fixed value of $H / D_{p}$. Thus, for smaller particles, the fourth thermocouple lies outside the measurement volume and is ignored in the analysis. When all four thermocouples are within the volume, the quadratic fit is made by choosing three of the four available measurements.

Using (2.3.2-5), equation (2.3.1) is readily solved with the boundary conditions $(2.3 .2)-(2.3 .5)$ by a standard separation-of-variables technique to obtain:

$$
\begin{aligned}
T(x, y)= & \sum_{n=1}^{\infty}\left[\frac{\sinh \frac{n \pi y}{L}}{\sinh \frac{n \pi H}{L}} \eta_{3}+\frac{\sinh \frac{n \pi(H-y)}{L}}{\sinh \frac{n \pi H}{L}} \eta_{1}\right] \sin \frac{n \pi x}{L}+\sum_{n=1}^{\infty}\left\{\frac{\sinh \frac{n \pi x}{H}}{\sinh \frac{n \pi L}{H}}\left[\eta_{2}-\frac{2 S U H^{2}\left(1-(-1)^{n}\right)}{V k(n \pi)^{3}}\right]\right. \\
& \left.+\frac{\sinh \frac{n \pi(L-x)}{H}}{\sinh \frac{n \pi L}{H}}\left[\eta_{4}-\frac{2 S U H^{2}\left(1-(-1)^{n}\right)}{V k(n \pi)^{3}}\right]+\frac{2 S U H^{2}\left(1-(-1)^{n}\right)}{V k(n \pi)^{3}}\right\} \sin \frac{n \pi y}{H}
\end{aligned}
$$

where: 


$$
\begin{aligned}
& \eta_{1}=\frac{2}{n \pi}\left[a_{1}-\left(c_{1} L^{2}+b_{1} L+a_{1}\right)(-1)^{n}\right]-\frac{4 c_{1} L^{2}}{(n \pi)^{3}}\left[1-(-1)^{n}\right] \\
& \eta_{2}=\frac{2}{n \pi}\left[a_{2}-\left(c_{2} H^{2}+b_{2} H+a_{2}\right)(-1)^{n}\right]-\frac{4 c_{2} H^{2}}{(n \pi)^{3}}\left[1-(-1)^{n}\right] \\
& \eta_{3}=\frac{2}{n \pi}\left[a_{3}-\left(c_{3} L^{2}+b_{3} L+a_{3}\right)(-1)^{n}\right]-\frac{4 c_{3} L^{2}}{(n \pi)^{3}}\left[1-(-1)^{n}\right] \\
& \eta_{1}=\frac{2}{n \pi}\left[a_{4}-\left(c_{4} H^{2}+b_{4} H+a_{4}\right)(-1)^{n}\right]-\frac{4 c_{1} H^{2}}{(a \pi)^{3}}\left[1-(-1)^{n}\right]
\end{aligned}
$$

Note that $k$ is still an unknown but may be determined by realizing that the material must absorb the heat supplied by the primary heater using the temperature gradient given by the above solution. I.e:

$$
\left.\frac{\partial T}{\partial y}\right|_{y=0}=-\frac{Q_{\text {wall }}}{A_{0} k} \quad \text { for } L_{1} \leq x \leq L_{2}
$$

where $\left(L_{2}-L_{1}\right)$ is the width and $A_{0}$ the surface area of the primary heater and $Q_{\text {wall }}$ is the heat it supplies. Equations (2.3.6) and (2.3.7) may then be solved for the conductivity, $k$, yielding:

$$
k=\frac{\frac{4 S U H^{2}}{V} \sum_{n=1}^{\infty} \frac{\cosh \frac{n \pi L_{2}}{H}-\cosh \frac{n \pi L_{1}}{H}}{\sinh \frac{n \pi L}{H}}\left(\frac{1-(-1)^{n}}{(n \pi)^{3}}\right)-\frac{2 S U H\left(L_{2}-L_{1}\right)}{V} \sum_{n=1}^{\infty} \frac{1-(-1)^{n}}{(n \pi)^{2}}-\frac{Q_{w a l l}\left(L_{2}-L_{1}\right)}{A_{0}}}{\sum_{n=1}^{\infty}\left(\frac{\eta_{3}}{\sinh \frac{n \pi H}{L}}-\frac{\eta_{1}}{\tanh \frac{n \pi H}{L}}\right)\left(\cos \frac{n \pi L_{1}}{L}-\cos \frac{n \pi L_{2}}{L}\right)+\sum_{n=1}^{\infty} \frac{\cosh \frac{n \pi L_{2}}{H}-\cosh \frac{n \pi L_{1}}{H}}{\sinh \frac{n \pi L}{H}}\left(\eta_{2}+\eta_{4}\right)}
$$

Note that the above analysis assumes that the thermal conductivity is a scaler, which is clearly a questionable assumption. For example, Wang et al. (1989) have indicated that this is not the case for rotating particles. Furthermore, Campbell (1989) shows that the granular temperature is anisotropic, which, further indicates that the conductivity is not a scaler. However, despite the trouble of solving the two-dimensional problem, the results never differ by as much as the thickness of a data point from assuming one-dimensional conduction. This indicates minimal heat conduction in the $x$-direction. As symmetry dictates that there can be no conduction in the direction of now, one can be reasonably certain that the above yields a reasonably accurate measure of the heat conduction in the $y$-direction.

\subsection{RESULTS AND DISCUSSION}

Results of the apparent thermal conductivity and the apparent viscosity for $3.0^{\mathrm{mm}}$ glassbeads are 




(a)



(b)

Figure 2.4.1: (a) The apparent conductivity, $k$ and (b) the apparent viscosity, $\mu$ as functions of shear rate for $3.0^{\mathrm{mm}}$ glassbeads.

plotted in Figures 2.4.1a and 2.4.1b as a function of shear rate for various values of solid fraction, $\nu$. At any given value of $\nu$, both vary linearly with the shear rate. This immediately demonstrates a relationship between the two quantities and indicates that they must have similar micromechanicai underpinnings. Even more interesting is that both theoretical analysis and computer simulation bave shown that, in a simple shear now, the granular temperature varies as the square of the shear rate (see, for example, Campbell \& Brennen (1985) and Lun et al. (1984)). The same conclusion might also be had by simple dimensional analysis. Thus, as both the apparent thermal conductivity and the viscosity vary directly proportional to the shear rate, they vary proportional to the square root of the granular temperature - just as kinetic theory predicts that viscosity of a gas varies with the square root of the thermodynamic temperature. This indicates that the granular temperature governs the internal transport of both heat and momentum in a rapid granular now.

However, the comparison between Figures 2.4.1a and 2.4.1b also demonstrates a fundamental difference between the the transport of heat and momentum. The slope of the apparent conductivity in Figure 2.4.1a decreases with increasing solid fraction, and, at the largest solid fractions, the conductivity becomes almost independent of shear rate. Exactly the opposite behavior is observed for the apparent viscosity shown in Figure 2.4.1b in which the slopes steadily increase with solid fraction. 
This indicates that differences exist in the internal mechanisms that lead to the transport of heat and momentum (although, whatever the mechanism, it must still increase the transport rate proportional to the shear rate and consequently proportional to the square root of the granular temperature). Now, the internal transport of momentum is fairly well understood to occur in a combination of two modes. The collisional mode accounts for the momentum which is transferred almost instantaneously between the centers of particles during a collision and the streaming mode describes the properties carried by particles as they follow their random walk through the bulk $m$ sterial. Obviously, the collisional mode dominates at dense packings where collisions are frequent and the streaming mode prevajls at dilute packings whare particles travel great distances between collisions. Note that, as the granular temperature both controls the speed at which the particles follow their random walk as well as the strength and frequency of collisions, it, therefore, controls the overall transport rate regardless of the transport mode. Now it is clear that heat, as well as momentum, can be transported in the streaming mode; i.e. as a particle follows its random walk through the material, it carries its heat as well as its momentum with it. But heat transfer is a relatively slow process and it is unlikely that any significant amount of heat will be transferred between particles during the brief duration of a collision. (This intuitive notion is confirmed by the analysis of Sun \& Chen (1988)). Consequently, there is no analog to the collisional mode in the internal mechanisms of thermal transport and it is reasonable to expect the streaming mode is the dominant heat transfer mechanism within a rapidly sheared material.

The dominance of the streaming mode of heat transport accounts for the difference in the behavior of the viscosity and thermal conductivity shown in Figure 2.4.1. At large concentrations, the collision rate will be large, but the movement of particles is very restricted. Consequently, one anticipates a large degree of momentum transport in the collisional mode, but a reduced degree of transport of both momentum and heat in the streaming mode. The former is apparent in the increase of the slope of the viscosity lines with concentration in Figure 2.4.1b. However, the corresponding restriction of particle movement reduces the effectiveness of the streaming component. Regardless of the magnitude of the granular temperature, (i.e. the magnitude of the shear rate) there will no streaming momentum transport if the particles cannot move. Consequently, the larger the solid concentration, the smaller the shear induced augmentation of the thermal conductivity, and, at the largest concentrations, the particles are essentially locked in position and the shear rate has almost no effect on the thermal conductivity. This process may be clearly seen in Figure 2.4.1a. Hunt (1990) has performed a theoretical analysis based on this same physical picture and predicts qualitatively similar results.

To check the scaling of the thermal conductivity, measurements were made for two other sizes of glassbeads, $1.9^{\mathrm{mm}}$ and $3.75^{\mathrm{mm}}$ in diameter and for steel shot of $2.2^{\mathrm{mm}}$ in diameter. The results of the 


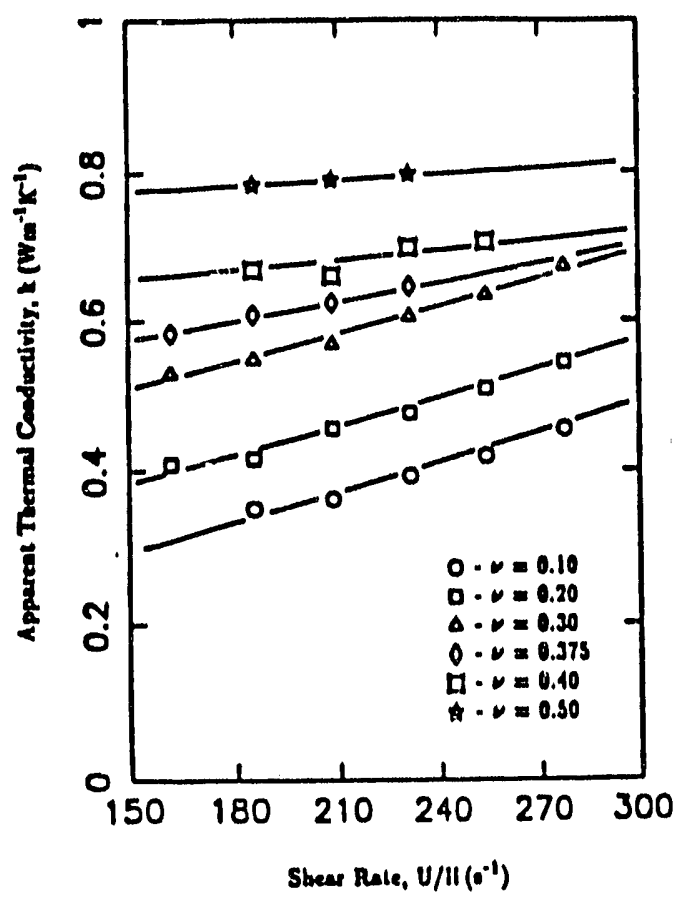

(a)

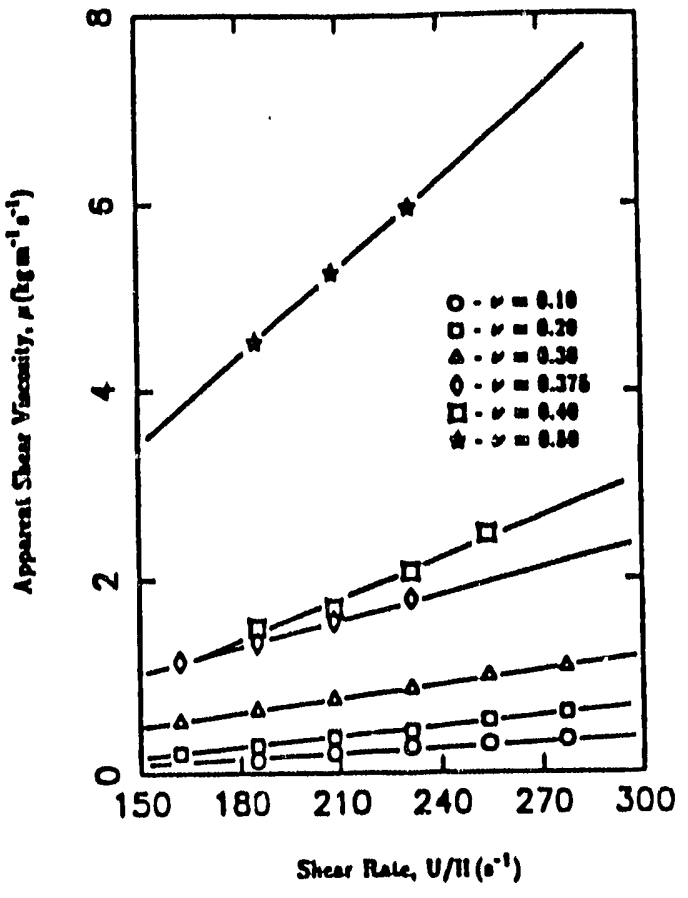

(1)

Figure 2.4.2: (a) The apparent conductivity, $k$ and (b) the apparent viscosity, $\mu$ as functions of shear rate for $1.9^{\mathrm{mm}}$ glassbeads.

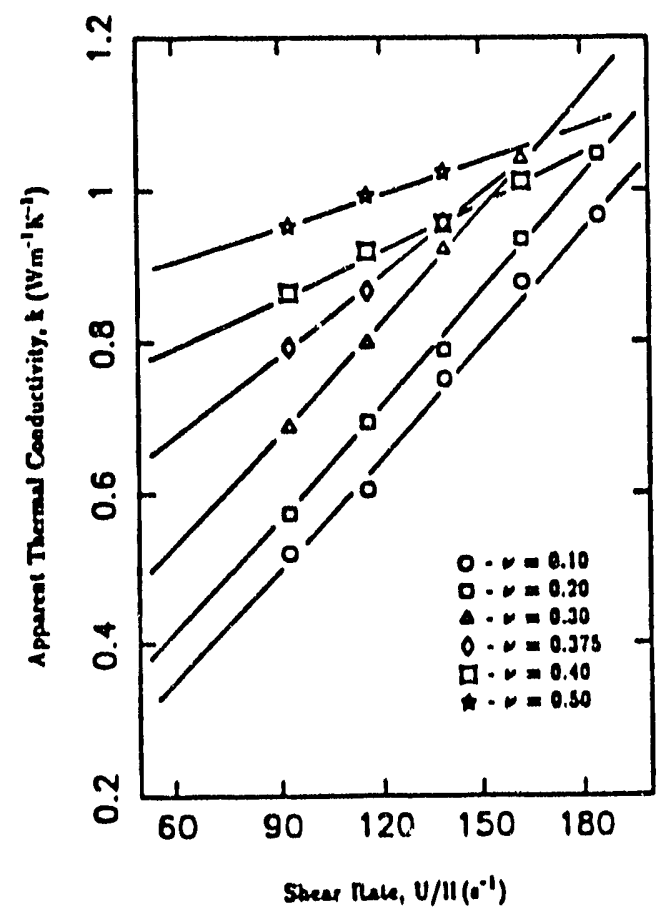

(a)

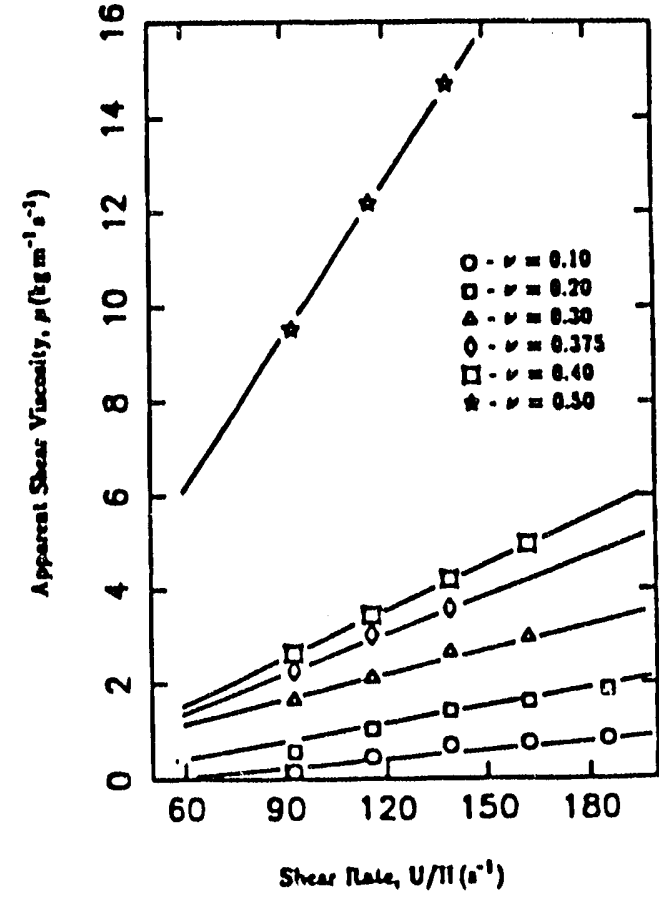

(B)

Figure 2.1.3: (a) The apparent conductivity, $k$ and (b) the apparent viscosity, $\mu$ as functions of shear rate for $3.75 \mathrm{~mm}$ glassbeads. 


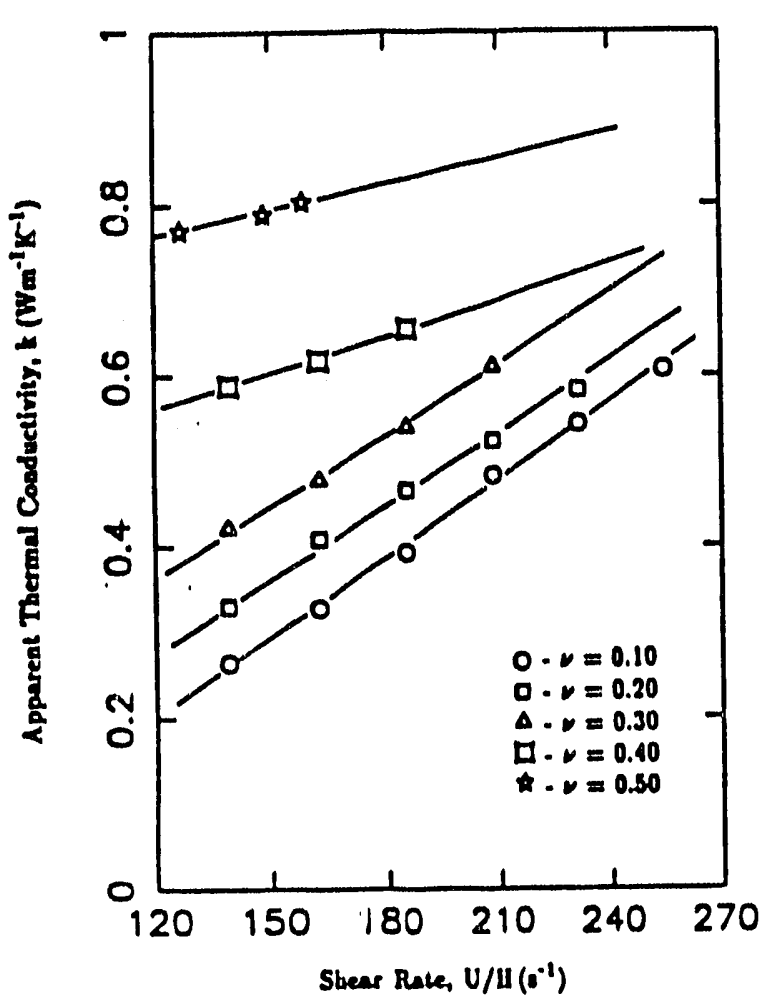

(a)

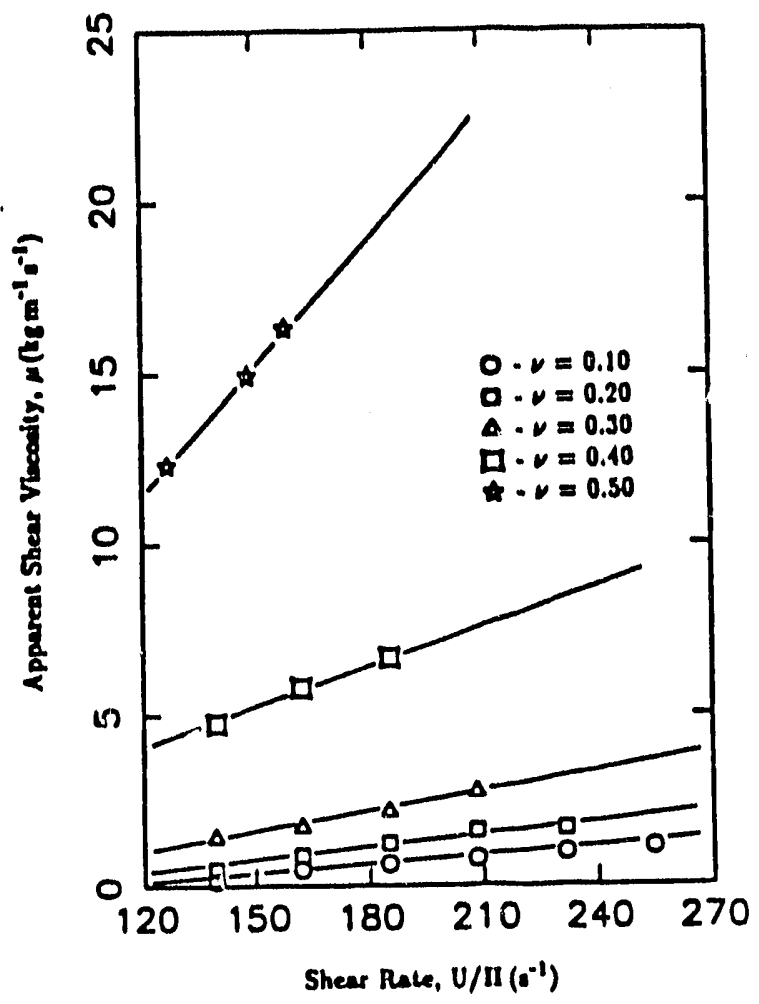

(b)

Figure 2.4.1: (a) The apparent conductivity, $k$ and (b) the apparent viscosity, $\mu$ as functions of shear rate for $2.2^{\mathrm{mm}}$ steel she'.

apparent thermal conductivity and the corresponding apparent shear viscosity measurements are shown in Figures 2.4.2-4 and display much the same behavior as the $3.0 \mathrm{~mm}$ glassbeads shown in Figure 2.4.1. As the apparent conductivities are shown to vary linearly with the shear rate for each material, density, and particle size, they can be fitted by a least squares fit into the form:

$$
k=k_{0}+b_{k} \frac{U}{H}
$$

The values of the constant, $k_{0}$, are plotted in Figure 2.4.5. Apparently, $k_{0}$ has questionable physical significance; in particular, it does not represent the conductivity of the test material in the limit of zero shear rate and, for some sets of the materials studied, even take on negative values at the smaller solid fractions. Furthermore, at larger concentrations, $k_{0}$, takes on values that are several time those that are predicted by packed bed correlations, such as those discussed in Xavier and Davidson (1985). However, for each material, $k_{0}$ increases with solid fraction, indicating that it may still reflects the increase in apparent conductivity of a static material as the solid fraction is increased. Collectively, 


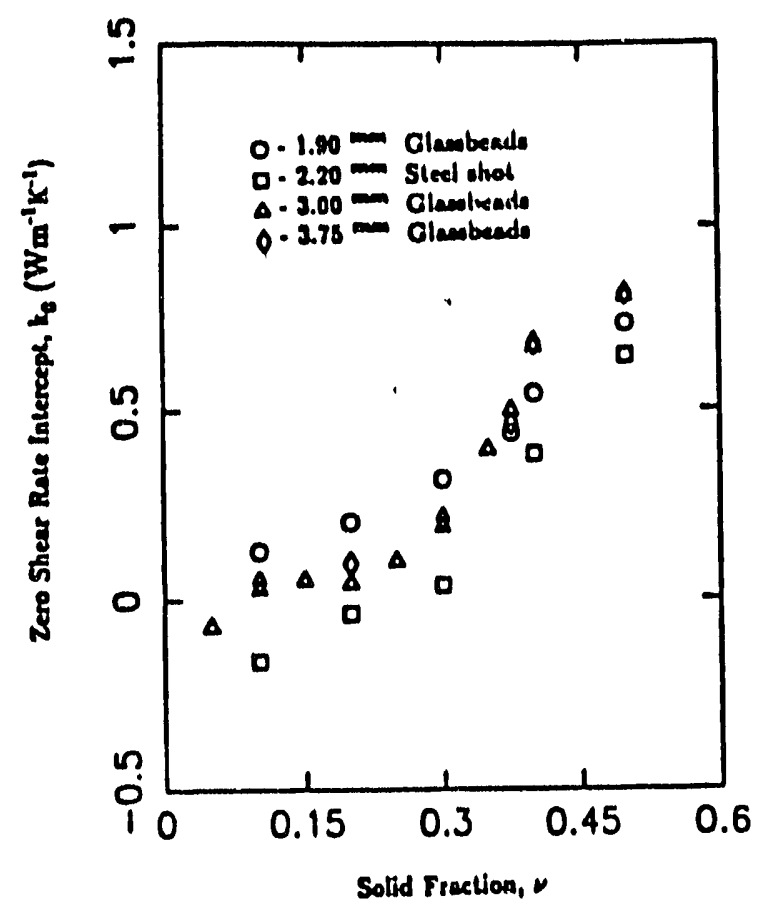

Figure 2.1.5: The zero shear rate intercepts, $k_{0}$, for the conductivity measurements, plotted as 1 function of the solid fraction, $\nu$.

this all indicates that the relationship between the thermal conductivity and the shear rate is not universally valid down to zero shear rates. Such behavior should not be surprising as the concept of a granular temperature, so important to the physical discussion presented above, is only appropriate for shear rates that are large enough so that the stresses applied to the bulk material can be supported by the inertia of the particles, (in much the same way as the pressure of a hard sphere gas is supported by the inertia of the molecules). At small shear rates, the bulk material behaves more like a solid than a nuid and it is impossible to induced a uniform shear rate on a material. Instead, most of the material will not deform and relative motion between particles will only be evident on thin slip lines called shear bands. Under such conditions, one would be surprised if the same behavior at low values of shear rate was apparent in the thermal conductivity as at high shear rates.

Since the principle internal mechanism oi heat transport is by the streaming mode, i.e. the heat carried by the particles as they follow their random paths through the bulk material, one expects that the apparent conductivity should scale with the heal capacity of the particle and thus be proportional to $\rho_{p} c_{p}$. As the thermal conductivity scales with the shear rate, $\mathbf{U}$, it follows then that the augmentation of the apparent conductivity is proportional to $\rho_{p} c_{p}\left(\frac{U}{H}\right)$, which bears the units of the thermal conductivity multiplied by (length) ${ }^{-2}$. As the particle diameter, $D_{P}$ is the only reasonable choice for the characteristic length scale of microscopic transport processes, an appropriate 
dimensionless form of the thermal conductivity is:

$$
\frac{k-k_{0}}{\rho_{p} c_{p} D_{p}^{2}\left(\frac{U}{H}\right)}
$$

which, dimensional analysis indicates can only be a function of the solid fraction, $\nu$. The resulting nondimensional conductivity enhancernent for all three sizes of glassbeads and steel shot, is plotted in Figure 2.4.6 as a function of the solid fraction, $\nu$, and, encouragingly, all of the data collapses onto a single curve. It should be pointed out that the success of this collapse is, in part, due to the fact that the steel and glassbeads have nearly the same coefficients of restitution (measured in drop tests to be about 0.8 and 0.75 respectively) and thus dissipate granular temperature at nearly the same rate. As a consequence, both materials will generate nearly the same granular temperature at the same shear rate. If the generated granular temperatures were significantly different, the internal transport rates would simultaneously reflect the difference in the granular temperature and the difference in the specific heat of the particles and as simple a scaling as (6.2) would not be possible.

An interesting feature of this curve is that it is nearly constant over a wide range of the smaller solid fractions and only begins to drop off at the larges: values of $\nu$. While this may seem surprising at first, it has a direct analogy in kinetic theory of gases in which the thermal conductivity of a perfect gas is found to be independent of the density. This occurs because the conductivity is a produ-t of the number density of heat carriers and the mean free path over which they travel. Increasing the density increases the number of carriers of heat, but, simultaneously, decreases the mean free path. At small concentrations, the mean free path is inversely proportional to the number density of carriers, so that their product and, consequently, the resulting thermal conductivity, is independent of concentration. A similar situation seems to be at work for the granular material studied here, even though a direct analogy cannot be made as there is no way to insure that the granular temperature, which governs the transport rate, is independent of concentration in this small $\nu$ region. A concentration dependence is visible only at very large solid fractions where the movement of particles is so restricted that they are only infrequently able to move past their neighbors. Under these conditions, one expects the mean free path to drop more rapidly than $1 / \nu$, so that the dimensionless conductivity goes to ze 0 . (This is analogous to the density correction in the Van der Waals equation of state.)

In much the same way as for the conductivity, the apparent viscosity, $\mu$ for each particle size, solid fraction and material can also be fitted by a least squares fit into the form: 


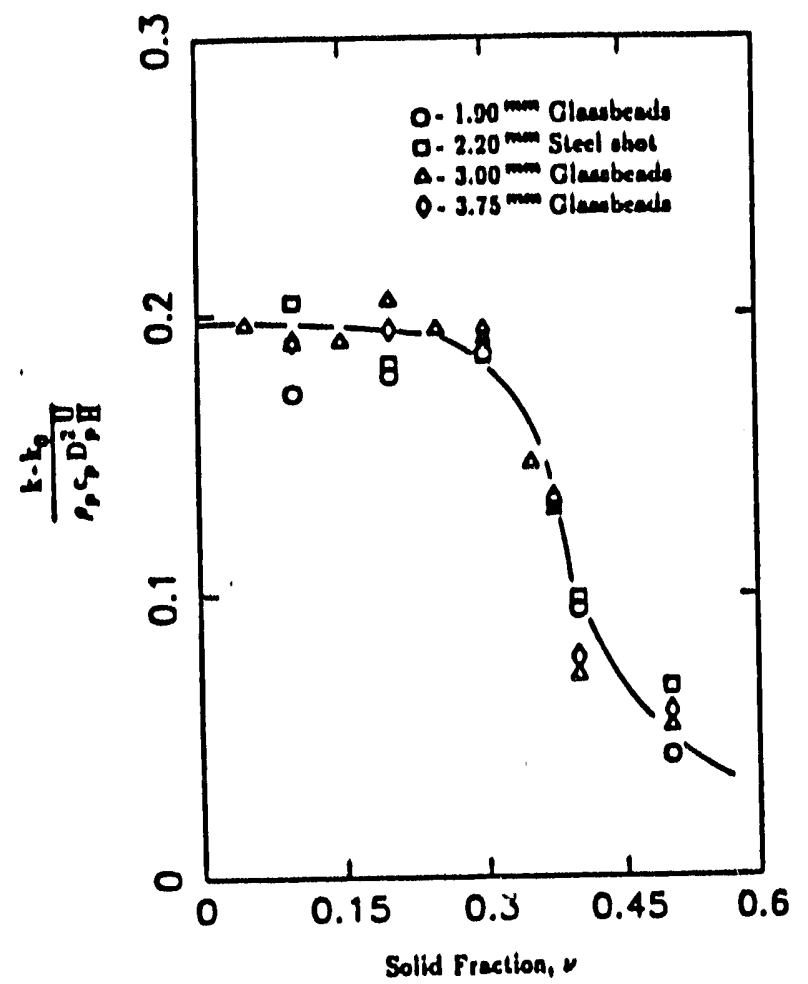

(a)

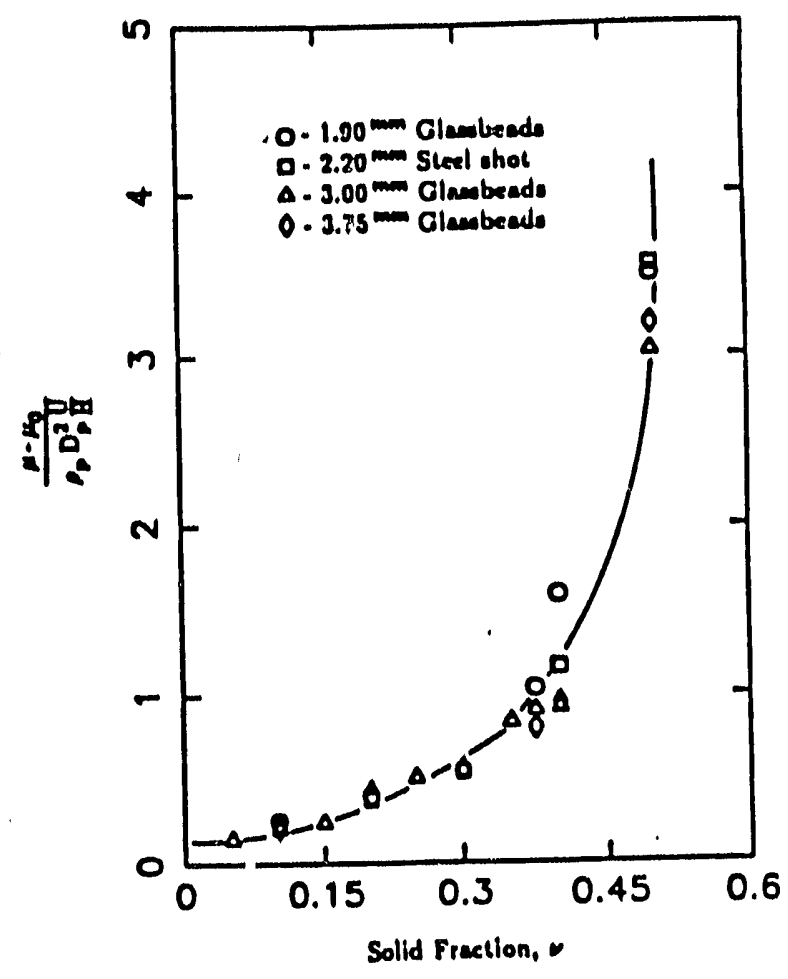

(b)

Figure 2.4.6: (a) Dimensionless conductivity augmentation as a function of solid fraction, $\nu$. (b) the corresponding dimensionless viscosity augmentation.

$$
\mu=\mu_{0}+b_{\mu} \frac{U}{\mathrm{H}}
$$

Here, all of the data extrapolates back to a non-zero value at zero shear rate for exactly the same reasons as described above for $k_{0}$, although the value of $\mu_{0}$ is generally small compared to that of $\mu_{\text {, }}$ while the value of $k_{0}$ is often comparable to that of $k$. By considering equation (1.1), a reasonable scaling of the viscosity is:

$$
\frac{\mu-\mu_{0}}{\rho_{p} D_{p}^{2}\left(\frac{U}{H}\right)}
$$

This dimensionless group is plotted in Figure 2.4.6b. As might be expected from the preceding discussion, this data asymptotes towards infinity at the larger values of the solid fraction. The asymptote represents the maximum concentration, beyond which, the material can no longer exhibit nuid-like behavior and, like a solid, will exhibit an infinite viscosity. 




Figure 2.1.7: Apparent $\mathrm{Prandtl}_{\text {number, }} \mathrm{Pr}=\left(\mu-\mu_{0}\right) c_{\mathrm{p}} /\left(k-k_{0}\right)$ as a function of solid fraction, $\nu$.

The ratio of the dimensionless groups given be equations (6.4) and (6.2) yields the apparent Prandtl number for the shearing mixturt:

$$
\operatorname{Pr}=\frac{c_{p}\left(\mu-\mu_{0}\right)}{k-k_{0}}
$$

Remember that the molecular and turbulent Prandtl numbers reflect the differences in the internal transport of heat and momentum. To make a direct analogy, this particular form of the Prandtl number given in (6.5) was chosen as, by subtracting away the zero intercepts, $\mu_{0}$ and $k_{0}$, it reflects the differences in the shear induced transport of beat and momentum that are the major topic of this paper. This Prandtl number is plotted in Figure 2.4.7 and can be seen to be a well defined function of the solid fraction, $\nu$. This indicates that the shear induced transport rates of heat and momentum are related through the solid concentration and a single material property, the specific heat of the solid phase, $c_{p}$. Remember that the majority of the beat is carried by particles, in the streaming mode, as they follow their random paths through the bulk material. Thus, the heal transport rate is proportional 
to the heat capacity of the particles which is equal to the mass of the particle multiplied by its specific heat, $c_{p}$, while the momentum transport is carried by the inertia of the particles and is therefore proportional only to the particle mass. Consequently, the dependence on mass disappears when taking the ratio that forms the Prandtl number, and leaves the specific heat as the sole remaining material property. The dependence on the solid concentration, $\nu$ reflects the fact that while both collisional and streaming modes contribute to the overall momentum transfer, only the streaming mode is important in the heat transfer process.

\subsection{CONCLUSIONS}

This paper has described measurements of the apparent thermal conductivity for a dry granular now while undergoing shear in an annular shear cell device. The results show that the bulk shear motion improves the internal transport of both heat and momentum and that both transport coefficients increase linearly with the imposed shear rate, indicating that similar internal mechanisms drive both transport processes. As the granular temperature varies as the square of the shear rate, the above results imply that both the conductivity and the viscosity enhancements are proportional to the square root of the granular temperature in much the same way as the kinetic theory of gases predicts that the viscosity and conductivity of a perfect gas are proportional to the square root of the thermodynamic temperature. This is a further indication that the granular temperature governs the internal transport processes in the rapidly shearing granular flows and fulrills much the same role as the thermodynamic temperature in a gas.

However, there are differences in the internal mechanisms that lead to the transport of heat and momentum. Momentum is transported both by particle collisions and by the random motion of particles through the bulk material. However, as heat transfer is a slow process, little heat can be conducted during the short duration of a collision and there is no analog of the collisional mode for the heat transport. Consequently, at large particle concentrations, when the particles have limited free range of motion, there is almost no shear induced enhancement of thermal conductivity. At the same time, the viscosity, working in the collisional mode, is asymptotically approaching infinity as the solid concentration approaches the maximurn value that can support a shear now.

By comparing the results of tests on particles of various sizes and thermal properties, it was demonstrated that the thermal conductivity varies as:

$$
k-k_{0}=\rho_{p} c_{p} D_{p}^{2} g(\nu) \frac{U}{H}
$$


where $g(\nu)$ is some function of the solid fraction, $\nu$. This provides the heat transfer analog to the famous prescription of Bagnold (1954) for the apparent viscosity:

$$
\mu-\mu_{0}=\rho_{p} D_{p}^{2} f(\nu) \underset{\mathrm{U}}{\mathrm{U}}
$$

where $f(\nu)$ is another function of $\nu$. Finally, we showed that the apparent Prandtl number for the shearing material is a function only of the solid fraction, further cementing the relationship between these two fundamental trassport properties. 


\section{CHAPTER 3: PARTICLE PRESSURE IN GAS FLUIDIZED BEDS}

The particle pressure may be thought of as the force per unit area exerted on a surface by the particulate phase of a multiphase mixture and, as such, reflects the total momentum transport that can be attributed to the motion of particles and their interactions. It has a direct analog in the kinetic theory of gases in which the pressure acting on a surface is visualized as a result of the impacts of molecules. The same picture can be applied to particle-fluid situations with the particles taking the place of molecules. The only difference between the two cases is that solid particles may, in addition to short-duration collisional impacts, transmit a force via long duration contacts with a surface. (For example, consider a mound of particles at rest in a gravitational field. Here, the particle pressure reflects the forces exerted across their contact points and increases nearly hydrostatically with depth. This is a very different picture than the impacts of thermal molecules, yet still represents a surface force within the material.)

A term involving particle pressures often appears in theoretical models of multiphase flows. Typically, such flows are modeled as individual phase equations, in which each phase, solid or fluid, is represented by its own set of equations, each with its own associated mass, energy, pressure. The Individual phase equations are then coupled together through interaction terms with the other phases in the mixture. So, in such a description of the motion of a solid-fluid mixture, one of the equations would describe the motion of the particulate phase and contain terms involving the particle pressure and other forces describing the interactions within, and the body forces acting on, the particle mass. Now, modeling the particle pressure term has always presented a problem. In extreme cases, some theorists have ignored it as physically unsound, others have taken it to equal the fluid pressure. However, neither of these arguments seems correct in light of the picture drawn in the last paragraph. Such extremely different viewpoints are excusable because there were no experimental measurements of particle pressure. Nonetheless, the behavior of the particle pressure can have significant effects on the behavior of multiphase systems. For example, Jackson (1985) and Garg (1975) have shown that the stability of a fluidized bed is affected by the dependence of the particie pressure on the void fraction, reflecting the possibility that instabilities may grow through the forces transmitted within the particle phase itself.

There are alternative pictures of the particle pressure that have grown out of the fluidized bed stability literature. The early stability theories (for example Murray (1965)) all indicated that a homogeneous fluidized bed should always be unstable, even though homogeneous nuidization has been experimentally obtained for beds composed of very fine particles. Many theorists have turned to the particle stress terms to try and account for this discrepancy. For example, Mutsers and Rietema (1977) envisioned the interparticle forces between small particles as being largely cohesive and, thus, able to 
resist the initial disturbances. Assuming that lubrication forces would keep particles from coming physically into contact, Batchelor (1988) conceived that the interparticle forces were transmitted across the intervening fluid and appeared as a viscous-like force that could damp out growing instabilities within the particle phase (although, in that analysis, the major contribution to stability came from a fluid particle interaction that forced the diffusion of particles). However, electrical conductivity data (e.g. Reed and Goldberger (1966) and Jones and Wheelock (1970)) indicates that particles do actually come into contact and form bridges that convey electric current across significant portions of the bed which, conceivably, could also transport interparticle forces.

As the particle pressure represents the forces applied by particles, its measurement may also be of importance in industrial processes. The obvious cases would be those processes that are concerned with attrition of particles where the attrition rate would be determined by the forces applied by other particles and by the wall - and thus should be reflected in the particle pressure. Furthermore, even if they do not cause particle breakage, the forces that particles experience may work harden their surfaces and change their physical, and possibly their chemical, attributes. Obviously, regions of largest particle pressure would be those that do the most damage and are either to be avoided or sought after depending on the desired outcome.

The only other attempt to measure the particle pressure is the recent study by Kumar et al. (1990) for liquid fluidized beds. They insertec a hydrophone into the wall of the bed and listened to the impacts of the particles. They calibrated their instrument by relating the output signal of the hydrophone to the impact velocity of test particles. Thus the primary measurement was the "thermal" velocity of the impacting particles and the particle pressure was inferred from the frequency and strength of these collisions. The results showed that, with increasing fluid velocity, the particle pressure first rose, reached a maximum and then fell. This reflects two competing processes internal to the material. Increasing the fluidizing velocity increases the agitation rate of the particles and consequently increases the strength of collisions. However, at the same time, it also decreases the density within the bed, and, with it, the number of particles collisions. Eventually, the number of collisions is reduced to the point that the pressure falls despite the increased strength of individual collisions. Kumar et al.'s experimental technique had several drawbacks. The first was that the probe calibration was very difficult and showed a great deal of scatter which, in turn, leads to a great deal of scatter in their results. Secondly, summing all the collisions on the hydrophone was very time consuming and, consequently, very few points appear in the data. Lastly, the hydrophone could only record the thermal-like motion of the particles and could not detect long duration contacts, and hence, could only determine a portion of the particle pressure. (This may also be an advantage as it allows the particle pressure to be decomposed into its constituent parts.) Such is not a problem for liquid-nuidized beds, 




Figure 3.1.1: A schematic of the particle pressure transducer.

but the same method would probably not work for gas-nuidized beds in which the particles are agitated en masse by the passage of bubbles. Still, it represents the only existent measurements of the particle pressure.

This chapter describes the measurement of particle pressure on the wall of a gas-fluidized bed using a probe that measures the sum of the particle and nuid forces and then cancels the nuid contribution. It can therefore measure the complete particle pressure in all the states of the fluidized bed, from the packed bed up to a fully slugging bed. The results are quite different than those measured by Kumar et al. (1990), a difference which most probably reflects the different behavior of gas versus liquid nuidized beds. A preliminary report of this work appeared in Campbell (1987); lowever, the probe design had not been refined at that time so that the results presented there are not entircly accurate.

\subsection{TUE PARTICLE PRESSURE TRANSDUCER}

The particle pressure is measured using a differential pressure transducer which is illustrated in Figure 3.1.1. Basically, the transducer consists of a diaphragm, nush mounted into the side wall of the nuidized bed, the front surface of which is exposed to both particle and gas forces. Small passages about the circumference of the diaphragm admit air, but no particles, through to the back side. Thus, the front of the diaphragm experiences both gas and particle pressures and the back side experiences only gas pressure, so that the net deflection of the diaphragm reflects the pressure exerted on the surface by particle interactions. Note, however, that due to the time it takes enough gas to pass from the front to the rear of the diaphragm, the instantaneous diaphragm deflection will not necessarily correspond to the instantaneous particle pressure. However, it can be easily seen by temporal averaging 
the equation of motion of a diaphragm, that the avezage displacement of the diaphragm will correspond to the average net force exerted on the surface. Thus, the signal from the pressure transducer is averaged over long time periods to yield the average particle pressure.

Preliminary experiments showed that the placement of the fluid ports surrounding the diaphragm could be critical. In a quiescent nuid, this does not present a problem as the fluid pressure is uniform across the face of the probe. Difliculties arise, however, if there is a pressure gradient tangential to the diaphragm surface (exactly as there would be if the probe were mounted in the side wall of a nuidized bed). In that case, nuid would tend to enter a pressure port where the pressure is large and leave through another pressure port where the pressure is lower, inducing a flow through the cavity behind the diaphragm. This could present a particular problem when the external pressure drop is large (as it would be if an airflow was driven through a bed of particles) so that the path through the transducer is easier than that on the outside. To avoid this difficulty, the current version of the transducer has only two pressure input ports located on opposite sides of the diaphragm along a diameter oriented perpendicular to the pressure gradient. In this way, the pressure difference between the ports is zero and no flow is induced across the interior of the transducer.

The design of the probe has gone through many iterations. The current probe is constructed of stainless steel and has a $3.8 \mathrm{~cm}$ diameter by $0.02 \mathrm{~cm}$ thick diaphragm. The diaphragm and its mounting structure were machined out of a solid piece of stainless steel. (This is not necessarily the best or the easiest way to manufacture such a probe, but it seems to be the best choice given the shop resources available.) The displacement of the diaphragm is measured by a MTI Accumeasure capacitance probe with a $0-0.13 \mathrm{~mm}$ range, although the displacement could also be measured by mounting strain gauges or by other means. The diaphragm is thickened directly in front of the displacement transducer so as to always presert a flat surface to the transducer. The holes that permit the passage of gas to the back side of the diaphragm were covered with a fine screen to keep them clear of particles. During the experiments on the nuidized bed, the signal from the probe was sampled by a Scientific Solutions Labmaster data acquisition card mounted in an IBM AT computer and averaged over many bubbling periods. (Samples take from 45 to 300 seconds to converge to stable averages of the particle pressures.)

\subsection{TESTING OF TIE PARTICLE PRESSURT' TRANSDUCER}

Unfortunately, there is no particle pressure standard against which to calibrate the probe. As these are the first particle pressure measurements, there is no way to create a known particle pressure and use it to evaluate the behavior of the probe. Consequently, the basic calibration of the particle 


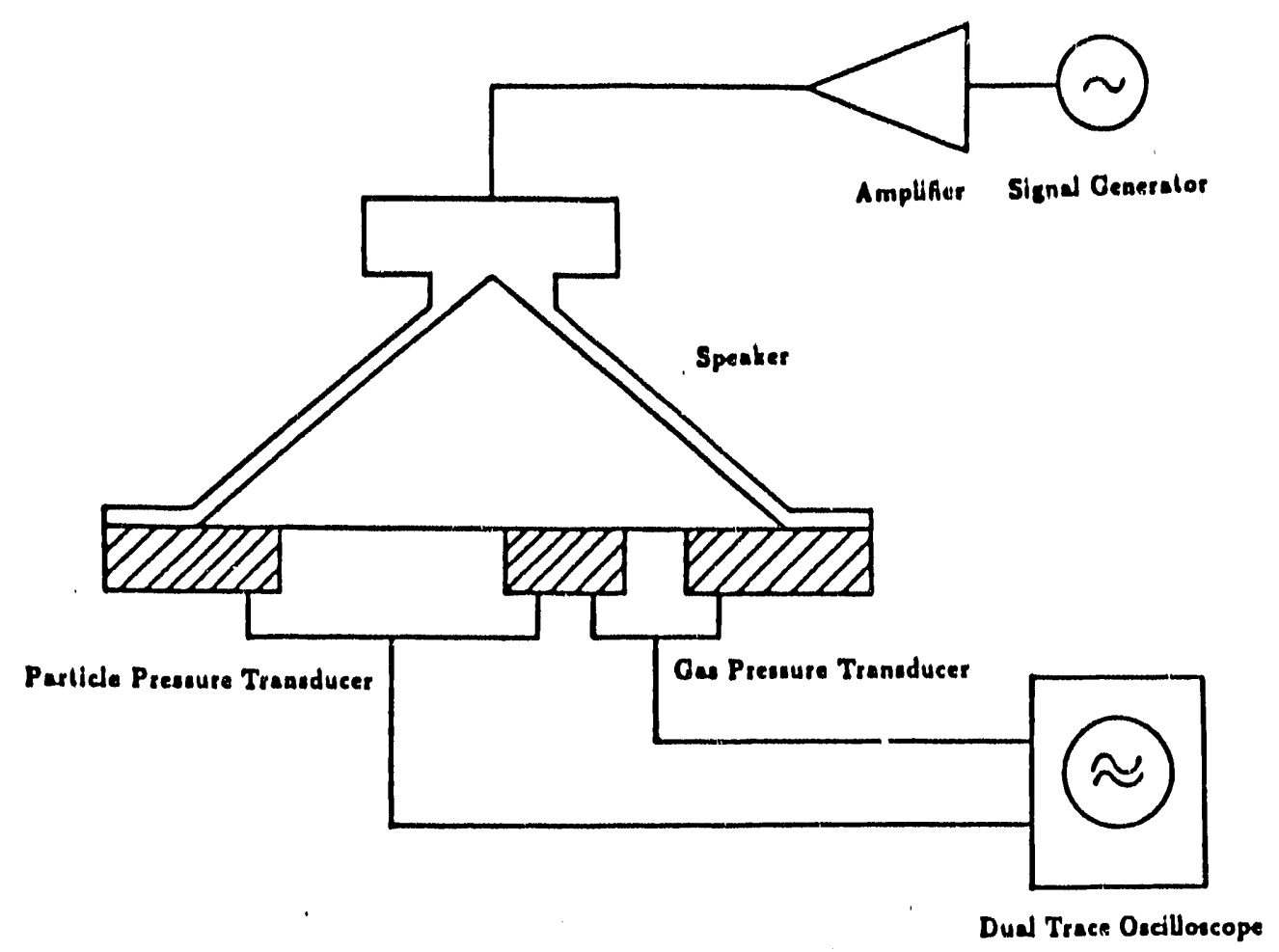

Figure 3.2.1: The experimental setup used to test the dynamic response of the particle pressure transducer

pressure transducer was performed in a static water column in much the same way as would be employed for a conventional nuid pressure transducer. A thin latex sheet was used to cover the gas ports and then the transducer was covered with various depths of water to simulate a static pressure loading. The output from the capacitance probe was found to vary linearly with the applied pressure.

The static calibration is somewhat troubling because it is conceivable that the particle pressure transducer design is dynamically very active. First of all, the diaphragm behaves as a circular plate pinned at the edges and consequently possesses natural modes of motion. But also, as mentioned previously, it is not expected that the transducer will react to the instantaneous particle pressure due to the time delay for the gas pressure behind the diaphragm to equilibrate to that in the gas outside. Because of this, one might consider the gas pressure behind the diaphragm as the response of an equivalent RC low pass filter. The resistance to air motion is imposed by the pressure drop in the gas moving through the narrow channels, the protecting screen and the particle mass outside as it enters or leaves the chamber behind the diaphragm. The capacitance is imposed by the volume behind the diaphragm that determines the quantity of gas that must be moved through the resistance to change the pressure. As an RC circuit acts as an analog averaging circuit, the higher the imposed frequency, the smaller the amplitude of the pressure nuctuation that is induced behind the diaphragm. But also, the capacitance induces a phase shift into the signal due to the time it takes the pressure behind the 
diaphragm to equalize. One might, then, expect some interaction between the diaphragm motion and the $\mathrm{RC}$ circuit that determines the pressure difference and it was not immediately clear that this will not have an effect on the output of the transducer. Therefore, the dynamics of the system had to be tested to be certain that the probe itself does not interfere with the particle-pressure messurement.

The system shown in Figure 3.2.1 was devised to test the dynamic response of the pressure transducer. A pressure source was provided by a standard audio speaker connected to a signal generator through an amplifier. An enclosed space was made by sealing the open end of the speaker with a nat plate. Into this flat plate were mounted the particle pressure transducer and a gas pressure transducer. The gas pressure transducer provided the reference signal for the pressure generated by the speaker which could then be compared to the output of the particle pressure transducer. The output from both systems was sent to a dual trace oscilloscope from which the relative magnitude of the two signals and the phase shift between them could be determined. Note, that as the particle pressure transducer ideally should not respond to changes in gas pressure, (at least in an averaged sense,) its time averaged output should be zero and indeed this was found to be the case. No non-zero time averaged output of the pressure transducer was measured for any of the test cases we were able to generate with this configuration. Thus, any output of the probe, under these conditions, is due to the time it takes the gas pressure behind the diaphragm to equilibrate to that outside.

The results of the relative amplitude and phase shift experiments are shown in Figures 3.2.2 and 3.2.3 respectively. When the test bed is fluidized, the particle pressure will come about from the bed motion induced by bubbles and slugs. It might then be surprising to notice that the range of frequencies shown here are really quite large compared to the bubbling and slugging rates that are observed in fluidized beds (which are iypically of the order of a Hertz). This is because at those low frequencies, (below about $10 \mathrm{~Hz}$ ) the output of the particle transducer is so small that it lies within the noise band of the system and cannot be discerned, indicating that the nuid pressure behind the diaphragm was capable of equilibrating the inside and outside pressures rapidly enough to keep up with the forcing signal. That was particularly encouraging as it showed that the transducer could be relied upon within the range of frequencies that is dominant in these experiments.

Figure 3.2.2 shows the ratio of the amplitudes of the peak-to-peak gas pressure and particle pressure outputs as a function of the driving frequency. The results show that, at $10 \mathrm{~Hz}$ the probe could equalize all but about $2 \%$ of the gas pressure and that the ratio $P_{\text {particle }} / P_{\text {sas }}$ increases monotonically with frequency from then on. The rise in the ratio reflects the slow approach towards a system resonance which occurs at about $500 \mathrm{~Hz}$ (well outside the range of this figure). Three different peak-topeak pressure amplitudes, 115, 230 and $460 \mathrm{~Pa}$, were studied. (As will be apparent in the following 




Figure 3.2.2: The ratio of the output of the particle pressure transducer to the applied gas pressure $\left(\mathrm{P}_{\text {particle }} / \mathrm{P}_{\mathrm{gas}}\right)$ as a function of the forcing frequency.

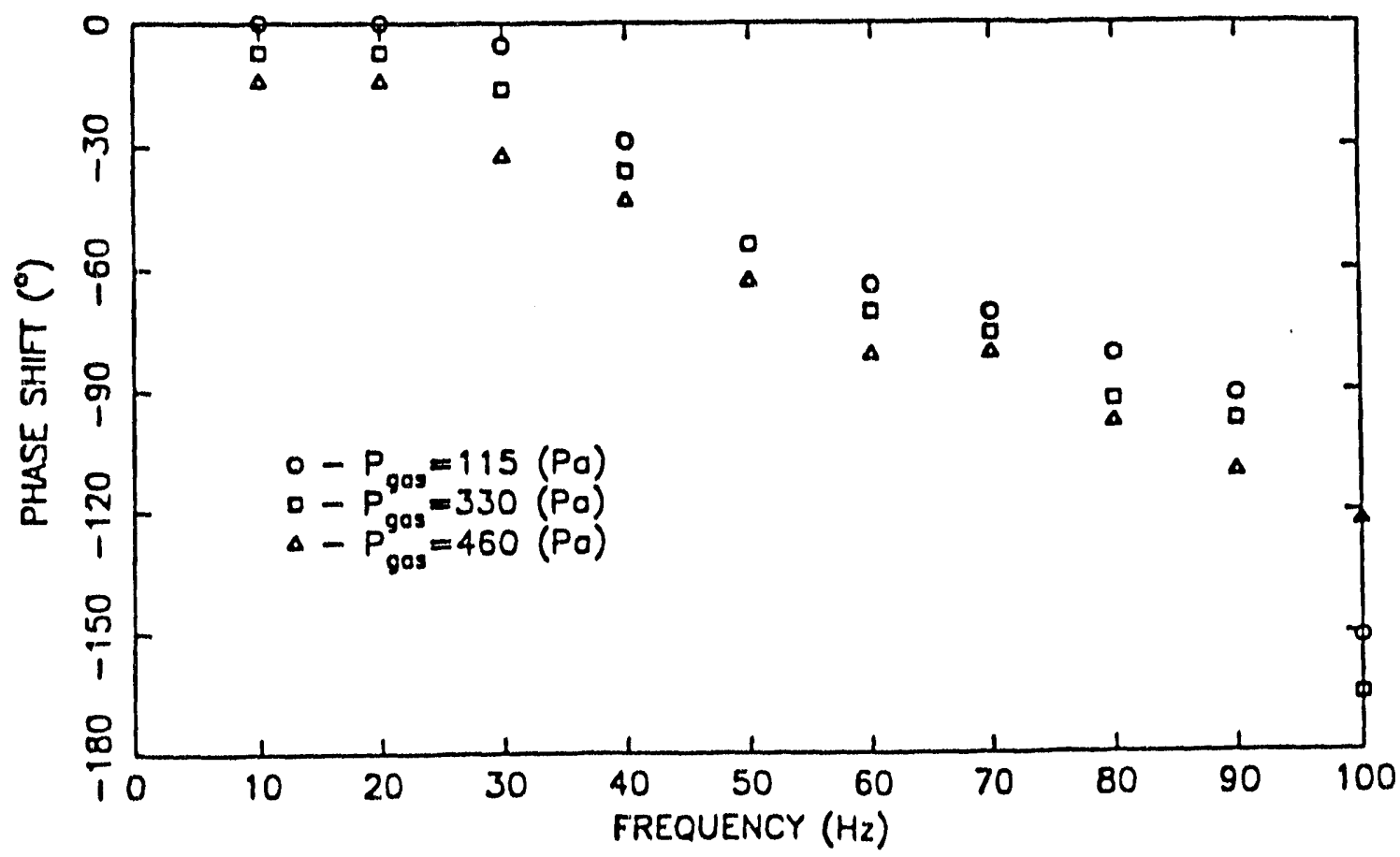

Figure 3.2.3: The phase shift between the particle pressure transducer's output and the applied gas pressure as a function of the forcing frequency. 


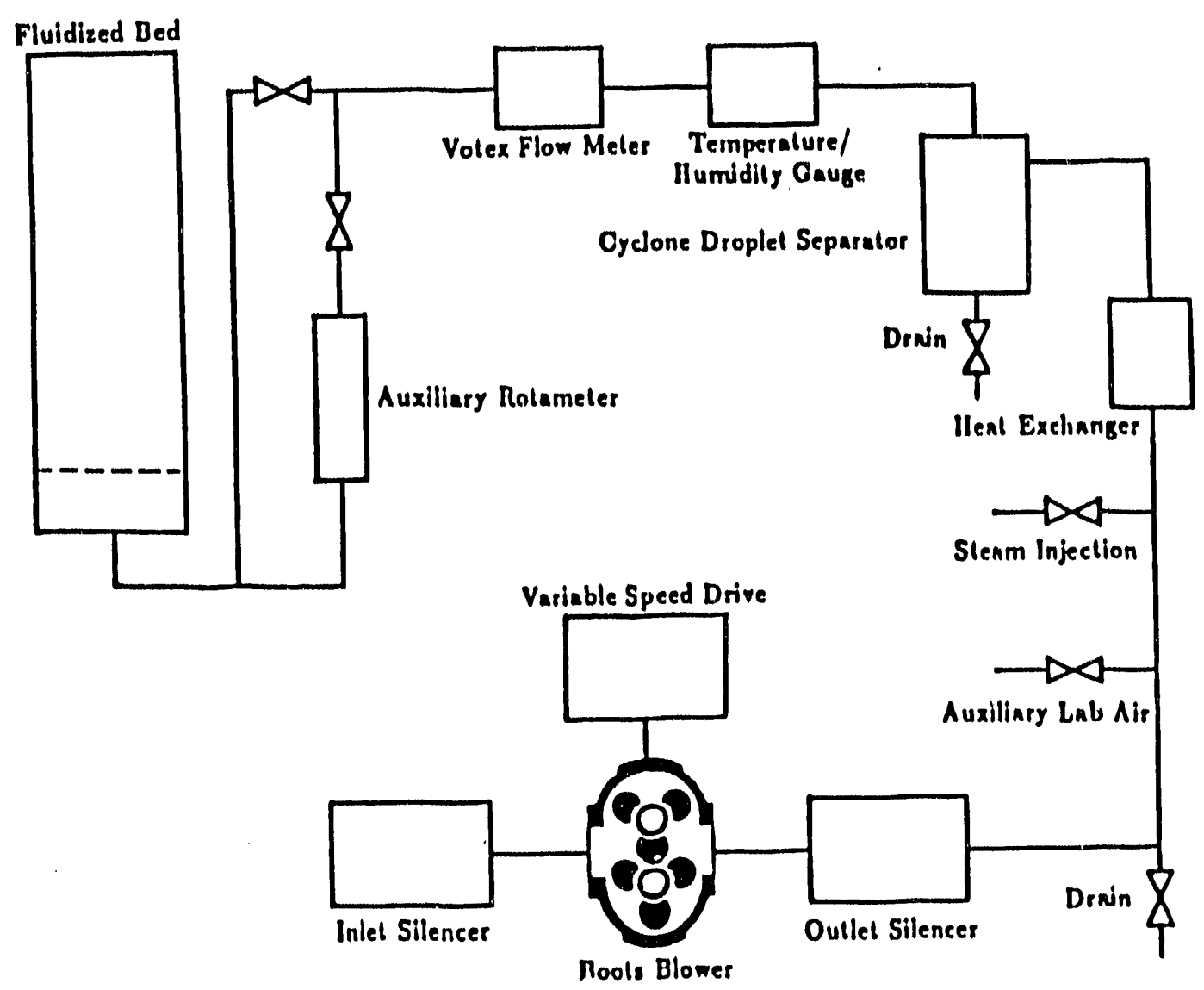

Figure 3.3.1: Schematic of the fluidized bed apparatus

sections, this covers the approximate range of average particle pressures observed in a fully fluidized bed.) A simple analysis indicates that the actual pressure difference across the diaphragm should not be a function of the applied pressure difference and, indeed this appears to be the case at low frequencies. An increase in the pressure ratio is apparent at high frequericies probably because the larger the applied pressure the more gas that must be moved across the ports and the higher the frequency, the less time there is available to complete the task. Figure 3.2.2 shows the phase shift between the imposed signal and the response from the particle pressure transducer. The phase shift can be scen to vary strongly with the applied pressure, varying at $10 \mathrm{IIz}$, from $0^{\circ}$ at $115 \mathrm{~Pa}$ applied pressure to about $15^{\circ}$ at $460 \mathrm{~Pa}$ applied pressure. The phase shift then drops monoto.lically as the frequency is increased. However, the strong effects occur at much higher frequencies than are likely to be encountered in nuidized beds, and, as the time-averaged output of the transducer is zero, there should be little or no eflect on the output of the transducer when it is used to measure the average particle pressures in gas-nuidized beds. 


\subsection{FLUIDIZED BED}

The experiments are performed in the gas-fluidized bed shown schematically in Figure 3.3.1. The bed is a $12.7 \times 12.7 \mathrm{~cm}(5 \times 5 i n$.) square channel, $122 \mathrm{~cm}$ ( 4 feet) tall. The air enters at the bottom through a porous plate distributor and is vented to the atmosphere through a fine screen at the top of the channel. Several ports for the pressure transducer, located 7.6, 15.2, 30.5, 45.7 and $61 \mathrm{~cm}(3,6,12$, 18 and 24 inches) from the distributor plate, were drilled in the side wall to allow observation of any gradients of particle pressure. The gas pressure drop across the bed was monitored using a U-tube manometer attached to a pressure tap near tle bottom of the channel. The void fraction in the bed was determined by a small gamma ray densitometer which consisted of a $100 \mu \mathrm{Ci} \mathrm{Cs}{ }^{137}$ source, collimated into a $5 \mathrm{~cm}(2 \mathrm{in})$ diameter beam and detected by a $5 \times 5 \mathrm{~cm}(2 \times 2 \mathrm{in})$ Bicron scintillation crystal detector attached to a Bicron Labtech scaler. The nuidizing air is provided by an M\&D Pneumatics 3204 threelobe Roots type blower driven by a 5HP variable speed motor so that the nowrate may be metered by varying the speed of the blower. (An auxiliary source of air is also available from the building's 'compressed air supply.) Steam, from a modified pressure cooker can be added to humidify the air so as to eliminate static buildup in the material inside the bed. The now then travels through a heat exchanger to remove the heat of compression and a small cyclone type device that removes any droplets that may become entrained in the airstream from the steam injection. Afterwards, the air passes over a temperature and humidity monitoring gauge and through a Nice Instruments vortex nowmeter. As vortex nowmeters are inaccurate below a minimum nowrate, a provision has been made so that the fow may be redirected through a Dwyer RMC-SSV-123 rotameter.

\subsection{PARTICLE PRESSURES IN GAS-FLUIDIZED BEDS}

As a point of reference, Figure 3.4.1 shows the particle pressure measured in the side wall of a fluidized bed along with the gas-pressure drop across the bed, both plotted as a function of the superficial gas velocity. Note that, as it covers several decades, the particle pressure is plotted on a log scale while the gas pressure drop is plotted, in its familiar form, on a linear scale. These particular results were taken with the probe positioned $15 \mathrm{~cm}$ (6in) from the distributor in a $12.7 \mathrm{~cm}(5 \mathrm{in}$.) squarc nuidized bed. The test material was $1.2 \mathrm{~mm}$ glassbeads carried through an entire cycle of loading to maximum nuidizing velocity and then unloading the bed completely back to zero velocity. (The properties of this and all of the materials used in this study may be found in Table I.) With zero nuidizing gas flow, the particle pressure is large, as the entire bed is supported only across interparticle contact points. Turning on the fluidizing gas causes a drop in particle pressure (and a corresponding 


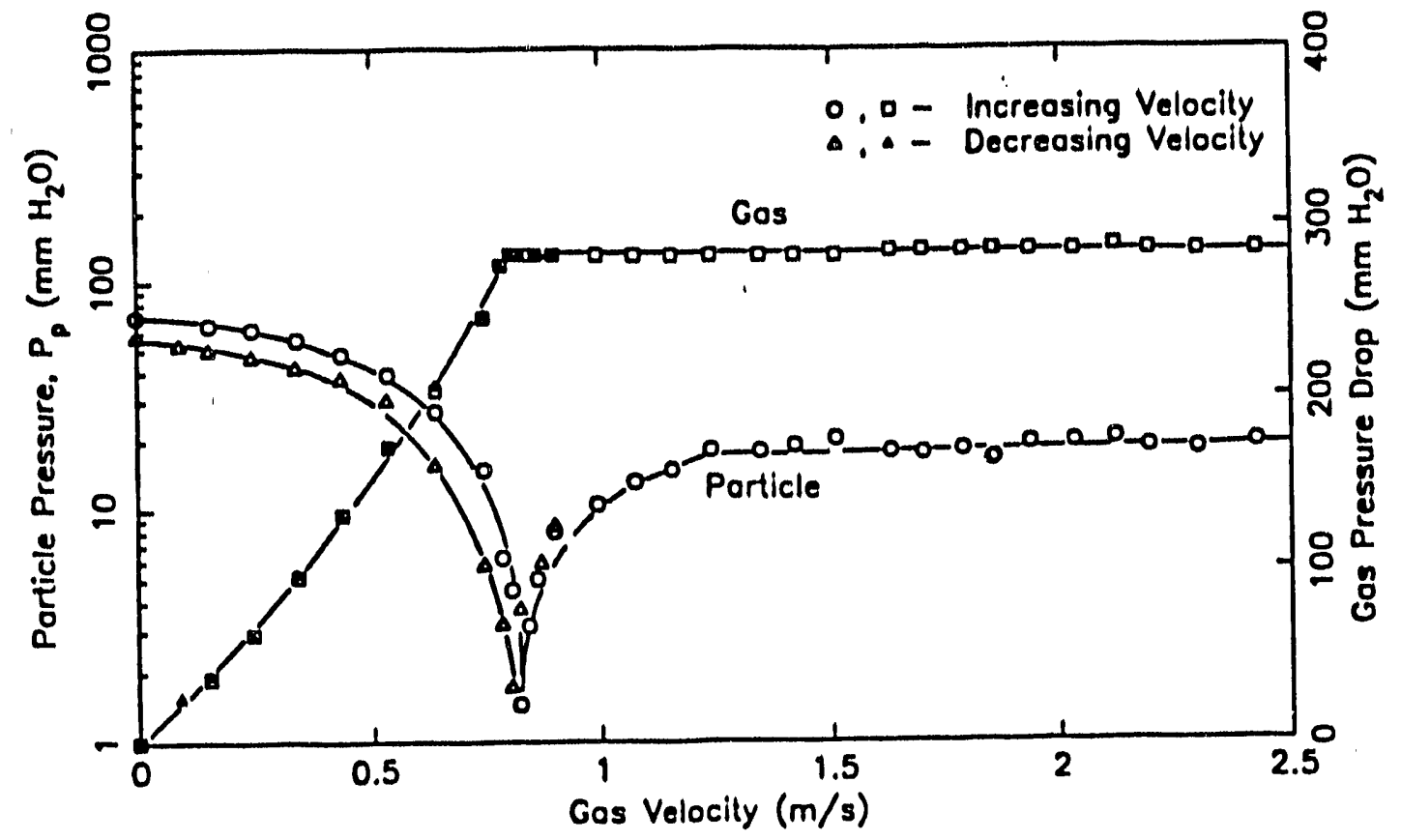

Figure 3.4.1: Particle pressure measurements on the sidewall of a gas-nuidized bed as a function of the superficial gas velocity. Also plotted is the gas pressure drop across the bed. The test material was $1.2 \mathrm{~mm}$ glassbeads with an initial bed depth of $21.6 \mathrm{~cm}$ and the probe was mounted $15.2 \mathrm{~cm}$ from the distributor. Note that the particle pressure is plotted on a log scale, while the air pressure drop is plotted on a linear scale.

increase in gas-pressure) as progressively more and more of the bed is supported by fluid forces. The particle pressure reaches a minimum, at just about the velocity where the bed is fully supported by the gas stream. A further increase in gas velocity causes the particle pressure to inciease once again presumably due to the agitation of the bed by bubbles. As the bed tegins to bubble almost immediately after minimum nuidization, the coincident rise in the particle pressure may be attributed to the agitation of the bed caused by bubble motion. But, towards the larger velocities, the bed begins to slug and, in this region, the particle pressure levels off and appears to approach a constant value.

TABLE I - TEST MATERIALS

\begin{tabular}{|c|c|c|}
\hline Material & Size $(\mathrm{mm})$ & Density $\left(\mathrm{kg} / \mathrm{m}^{3}\right)$ \\
\hline Glass Bcads & 0.50 & 2440 \\
\hline Glass Bcads & 1.20 & 2440 \\
\hline Glass Bcads & 2.80 & 2440 \\
\hline Steel Shot & 0.82 & 7600 \\
\hline Polystyrene Beads & 0.75 & 1050 \\
\hline
\end{tabular}


It is somewhat interesting that the particle pressure does not go to zero at its minimum value. The particle pressure exerted on the side wall of the bed should be some fraction of the total weight of the bed. When the bed is fully supported by nuid forces, it essentially has no weight and one might expect a zero particle pressure. However, this is not what is observed (although the measured values do approach the resolution limit of the pressure transducer). This discrepancy between intuition and observation may be attributed to a variety of causes. It is possible that, so near the fluidization point, the particles in the bed are beginning to show an almost thermal like agitation (much like that observed by Kumar et al. (1990)), which could offset some of the pressure reduction due to increasing gas flowrate. However, a more mundane possibility is that, as the particle pressure reaches its minimum at different velocities for different locations in the bed, (as will be shown later in Figure 3.4 .5 ,) the measured value may not reflect the actual minimum value obtained at that location but rather an average over the surface of the diaphragm.

Note that the location of this minimum provides a well-defined location that corresponds to the minimum fluidization velocity in the bed. Certainly, as it reflects the degree to which the bed is supported by nuid forces, it has much more physical significance than most methods of determining of minimum fluidization (which generally involve some sort of graphical analysis of the gas pressure-drop curve.) However, all of the results in this paper use relatively large particles for which the minimum nuidization point and the bubbling point coincide, and it is not clear what would happen for smaller particles which have an intermediate stage where they fluidize without bubbling. Therefore, in this paper, as the particle pressure will usually be plotted by itself, without the corresponding gas pressuredrop line, we will use the location of this minimum as a reference point for minimum nuidization. Points to the left of the minimum will be referred to as the packed bed state and those to the right as the fluidized state.

Unloading the superficial gas velocity from its maximum value, causes the particle pressure to return along much the same path as it followed on loading. The path begins to deviate only near the point of minimum nuidization and, from that point on, returns along a lower path than it followed on loading in the region where the bed is fixed. At first, this caused great consternation that there might be hysteresis in the particle pressure probe, but ihis pattern of behavior is repeatable and an indication of a change in the internal structure of the packed bed. Interestingly enough, the bed returns at a smaller density and consequently a greater height. This means that a larger fraction of the bed resides above the particle pressure transducer so that, if the pressure were distributed hydrostatically, one would expect larger, not smaller, particle pressures. This implies that, on returning from a nuidized state, the forces within the bed are redistributed. The larger particle pressure, observed before turning on the fluidizing gas, indicates that a larger portion of the bed was supported by frictional resistance on 




Figure 3.4.2: Particle pressures measured at three locations along the side wall of the bed all of which lie within the initial packed bed. Notice that in the packed bed region, the particle pressure decreases going upward through the bed while in the fully-nuidized region, the particle pressure increases. The test material was $0.5 \mathrm{~mm}$ glassbeads with an initial bed depth of $43.2 \mathrm{~cm}$.

the side walls. The smaller particle pressure, observed on unloading the system, indicates that more of the bed weight is distributed vertically and supported on the distributor plate. (This may be an indication that the material may form internal vertical channels to ease the passage of gas through the packed bed.) Bowever, we noticed that tapping the side of the apparatus will cause the bed to settle, altering the internal distribution of forces and changing the path followed by the particle pressure. This indicates that the loading and unloading paths are not unique.

Figure 3.4.2 shows the particle pressure measurements for $0.5 \mathrm{~mm}$ glassbeads, with the probemounted at thrce different locations on the side wall of the bed: $7.6,15.2$ and $30.5 \mathrm{~cm}(3,6$ and 12 in.) from the distributor. In its packed state the bed was initially about $43 \mathrm{~cm}(17$ in.) deep. Before minimum nuidization, the deeper one goes in the bed, the larger the particle pressurc; i.e. the particle pressure is behaving as one would expect of a hydrostatic system: the particle pressure is larger at the $7.6 \mathrm{~cm}$ location (the deepest in the bed) than at the $15.2 \mathrm{~cm}$ location, which is, in turn, larger than at the $30.5 \mathrm{~cm}$ location. However, beyond the nuidization point, the situation reverses itself with the particle pressure increasing going upward through the bed. This may be understood by remembering that bubbles and slugs grow rapidly as they progress upward so that the higher the point in the bed the 


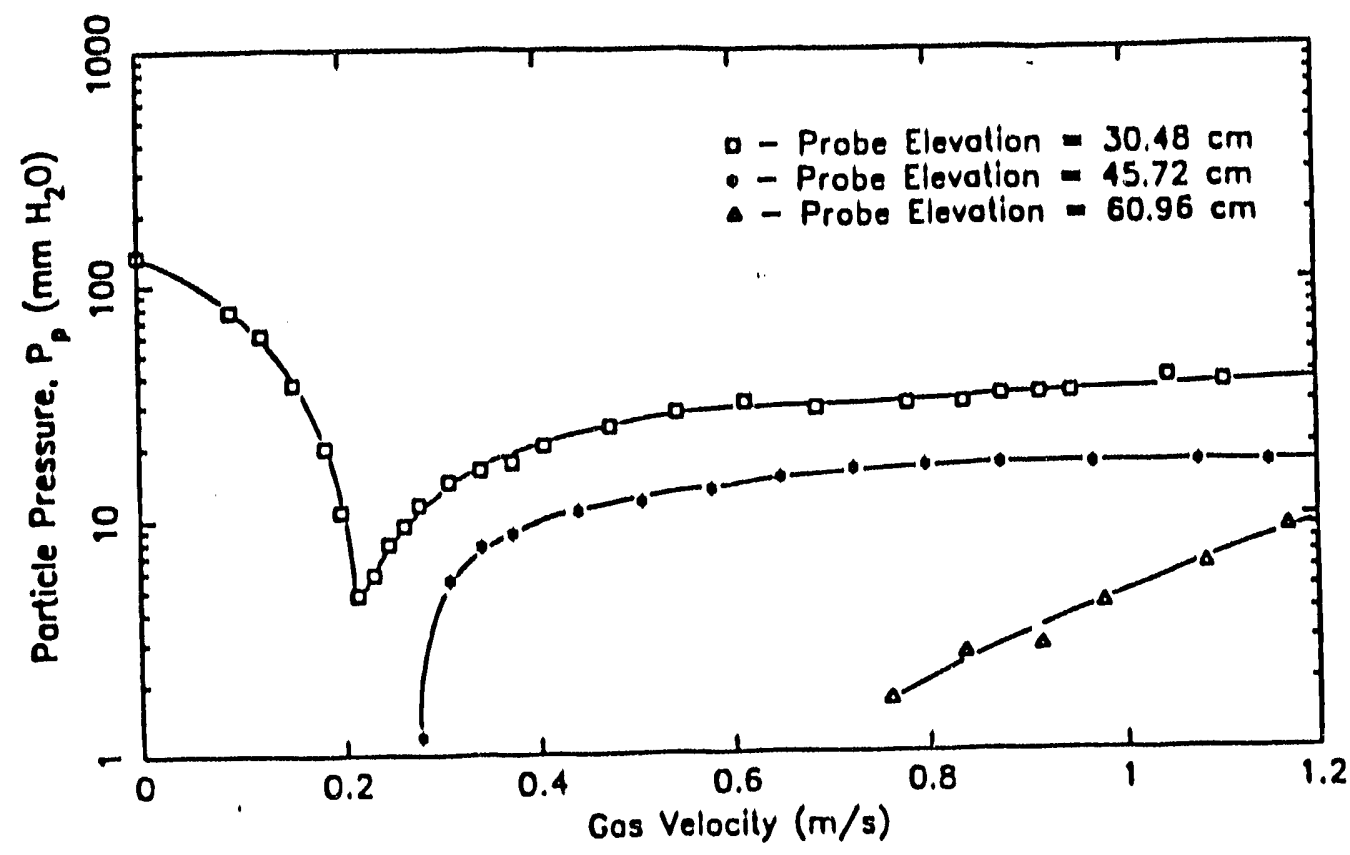

Figure 3.1.3: Particle pressures measured at two locations in the freeboard region above the original packed bed. For comparison, the curve from the $30.5 \mathrm{~cm}$ position is reproduced from Figure 4. Notice that the particle pressure decreases with height outside the original packed bed. The test material was $0.5 \mathrm{~mm}$ glassbeads with an initial bed depth of $43.2 \mathrm{~cm}$..

larger the particle agitation and the larger the particle pressure.

Now, all of the points shown in Figure 3.4.2 were taken with the probe location that lies within the initial packed bed (which was approximately $43 \mathrm{~cm}$ ( 17 inches) deep). Figure 3.4 .3 shows what happens when the probe is mounted at locations that are in the freeboard region outside the initial packed bed. Here the data for the $30.5 \mathrm{~cm}$ ( $12 \mathrm{in}$.) location is reproduced from Figure 3.4 .2 as a point of comparison to data taken with the probe mounted 46 and $61 \mathrm{~cm}$ (18 and $24 \mathrm{in}$.) above the distributor plate. At the 46 and $61 \mathrm{~cm}$ locations, the probe is initially uncovered so that the particle pressure iszero until the bed has expanded enough to cover he probe. In these cases, the particle pressures become smaller as the probe is moved upward. This should be expected, as when the bed is slugging, it will often collapse nearly back to the packed bed state, uncovering the probe. (Note, however, that the pressures experienced at the $46 \mathrm{~cm}$ location are still larger than those observed at the lowest $(7.6 \mathrm{~cm})$ position, shown in Figure 3.4.2.) In an averaged sense, it might be argued that the bed experiences an upward pointing particle pressure gradient inside the original packed bed and a downward pointing particle pressure gradient outside of it. 




Figure 3.4.1: The effect of bed depth at minimum nuidization on the particle pressure. The test material is $0.5 \mathrm{~mm}$ glassbeads and the probe was mounted $7.6 \mathrm{~cm}$ from the distributor.



Figure 3.1.5: An example showing the progression of the minimum point downward through the bed as the superficial gas velocity is increased. The test material is $1.2 \mathrm{~mm}$ glassbeads. Note that the particle pressure is plotted on a log scale, while the air pressure drop is plotted on a linear scalc. 
The effect of bed height is shown in Figure 3.4.4. (Note that the height of a fluidized bed increases with the superficial gas velocity and is thus not a well determined quantity; so, borrowing inspiration from the literature, we define the bed height at the minimum fluidization state.) One might expect that changing the depth of the bed over the probe position might have the same effect as mounting the probe at a lower position along the side wall. Part of this prediction appears to be true in that, within the packed bed region, increasing the bed height above the transducer does increase the the particle pressure. However, when the bed is fully nuidized, the particle pressure appears to be independent of the bed height. Thus, apparently, it is the probe's height above the distributor, and notthe depth of the bed above the probe, that affects the particle pressure in the fully fluidized region.

The distribution in particle pressures throughout the bed reveals some information about the state of the bed near minimum nuidization. This is apparent in Figure 3.4.2, but is easier to see with larger glassbeads as the response to increased gas velocity is slower so that the effect is spread out over a much wider range. Figure 3.4.5 shows particle pressure measurements about the initial nuidization point, for $1.2 \mathrm{~mm}$ glassbeads in a bed that is initially about $43 \mathrm{~cm}$ deep. This data was taken in three separate experiments, but great care was taken to prepare the beds in exactly the same way for each case. Note that the minimum point occurs at slightly higher velocities near the bottom of the bed than near the top. Furthermore, the value of the particle pressure at the minimum is smaller, the deeper one goes in the bed. Thus it appears that the bed does not reach this minimum value all at once but, instead, a front progresses down the bed over a small range of gas velocities. Most probably, this reflects the fact that bubbles first appear near the top of the bed and gradually progress downward towards the distributor with increasing gas velocity.

The next task is to try and understand the parameters - such as particle size and density - on which the particle pressure depends. As a first candidate, consider the effect of particle size demonstrated in Figure 3.4.6. This shows particle pressure measurements for three sizes of glassbeads, taken at the $15.2 \mathrm{~cm}$ probe location. As different particle sizes nuidize at different velocities, the curves would look vastly different if plotted against the nuidizing gas velocity. Instead, the particle pressure is plotted against the void fraction, $c$ as the value of the void fraction at minimum nuidization is nearly independent of particle size. (Note, however, that as the void fraction increases monotonically with gas velocity, an increase in 6 may be interpreted as an increase in the superficial gas velocity.) Plotted in this way, no significant effect of particle size can be seen, indicating that the magnitude of the particle pressures depends mostly on the material type. Note that there is scatter in the data, especially near minimum fluidization, but this may simply reflect small variations in the void fraction at minimum nuidization of the respective particle sizes. 


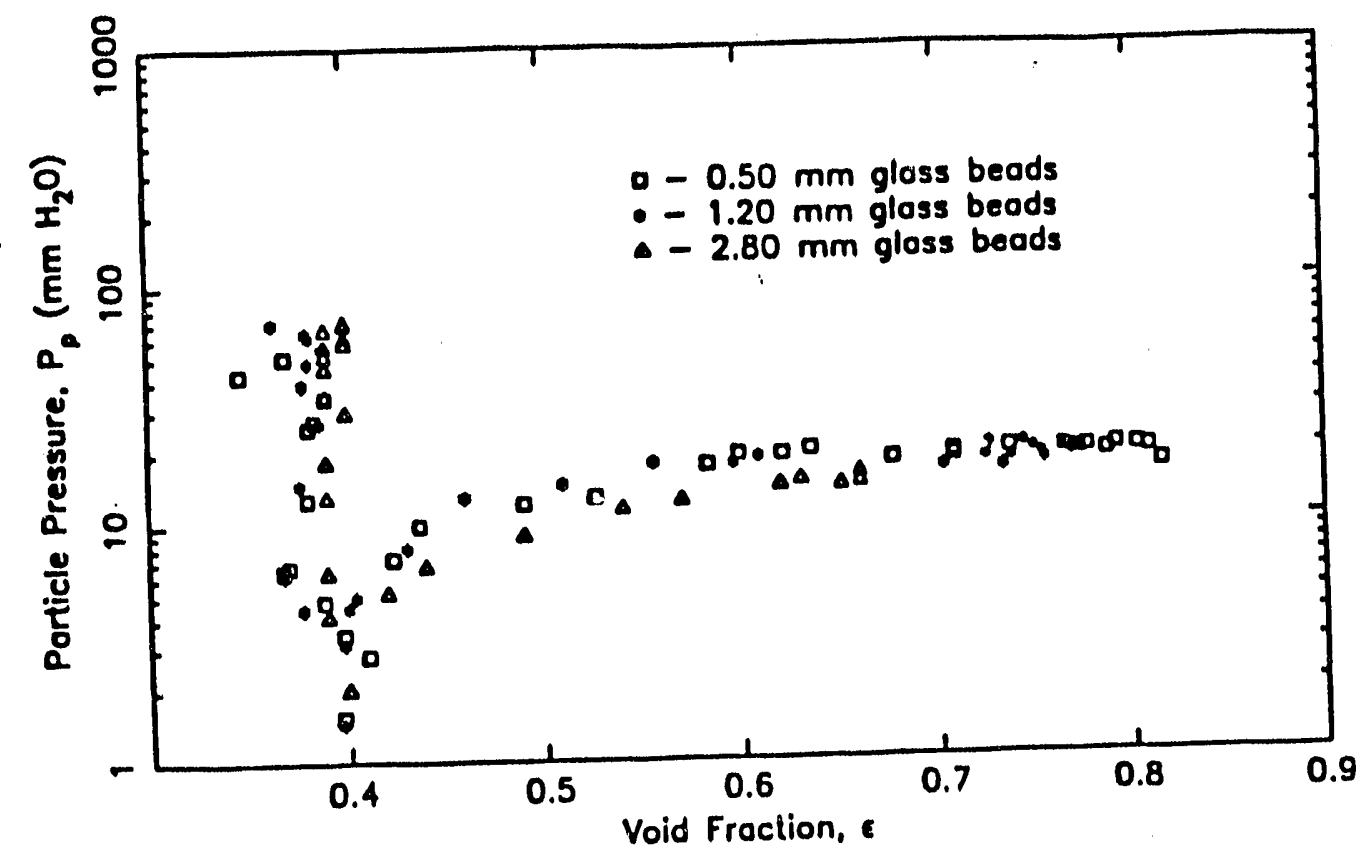

Figure 3.1.6: The effect of particle size on the particle pressure. This time, the data is plotted against the void fraction, $c$.

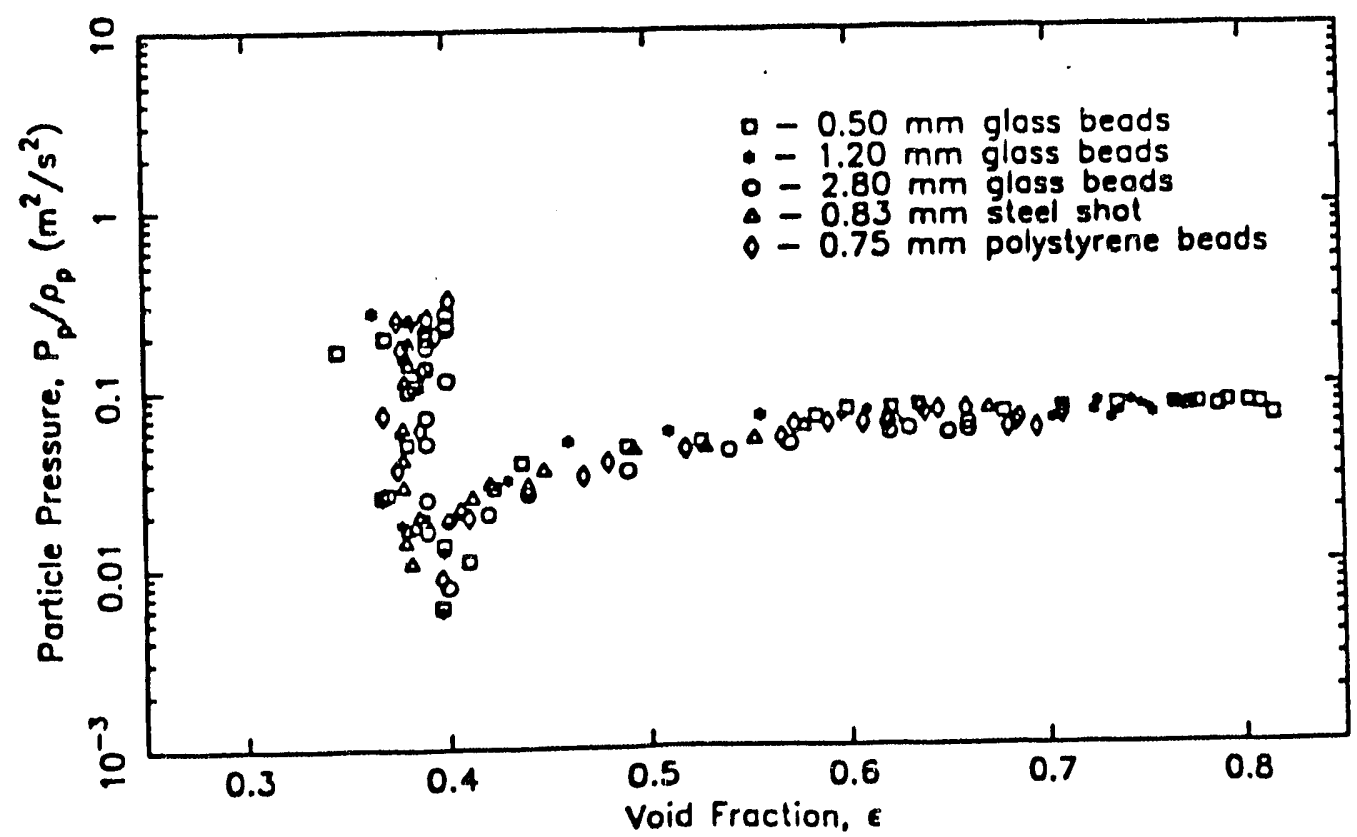

Figure 3.1.7: The effect of particle density on the particle pressure. Note that the particle pressurc scales with the density. 
Figure 3.4.7 shows the particle pressure measurements scaled by the solid particle density $\rho_{\mathrm{p}}$. Once agaia, this is plotted as a function of the void fraction rather than the superficial gas velocity as each material type will have vastly different minimum nuidization velocities. The results indicate that the particle pressure scales with the particle density. This is certainly true for the larger void fractions which correspond to the region where the bed is slugging, although, once again, there is variation near the minimum fluidization point probably due to the fact that the different materials fluidize at slightly different void fractions.

Figure 3.4.7 indicates that we are getting close in determining the dimensional scaling of the particle pressure. To make $P_{p} / \rho_{p}$ dimensionless, would require a grouping with units of velocity squared in the denominator. However, the missing quantity is clearly not the flow velocity squared as the velocities needed to nuidize the various materials and sizes are very different. It seems more likely that the grouping has the form $\mathrm{gL}$ where $\mathrm{g}$ is the gravitational acceleration and $\mathrm{L}$ is some appropriate length scale. Figure 3.4.6 shows that the length scale is not the particle size and Figure 3.4.4 indicates that it cannot be the bed height.

The indications are, however, that the particle pressures in the fully nuidized region are driven by the bubble motion. So far, we have shown that, the particle pressures increases moving upward through the initial packed bed, (Figure 3.4.2) but are independent of bed depth (Figure 3.4.4) and the particle size (Figure 3.4.6). All of this suggests that the proper length scale might be the bubble size as none of the correlations for bubble size indicate a dependence on the bed depth, the particle size or particle density except through the way they infuence the minimum fluidization velocity. Furthermore, bubbles and slug will grow as they move upward through the bed which could explain the dependence on probe position shown in Figure 3.4.2. Lastly, the progression of the minimum point downwards through the bed (Figure 3.4.5) may indicate that the minimum simply follows the initial appearance of bubbles at progressively deeper locations. Unfortunately, we had no means with which to measure the bubble size and had to depend on correlations published in the literature. We chose the correlation of Mori and Wen (1975) as it is the only correlation that included the effect of bed width. (As our bed was rather narrow, we expected some effect of bed width on the results.) They predict:

$$
D_{e}=D_{e, \infty} \cdot\left(D_{e, \infty}-D_{e, 0}\right) e^{-0.3 x / D}
$$

where:

$$
D_{e, 0}=0.376\left(U-U_{m}\right)^{2}
$$


and:

$$
D_{e, \infty}=1.49\left[D^{2}\left(U-U_{m s}\right)\right]^{0.4}
$$

Here, $D_{e}$ is the equivalent bubble diameter (i.e. the diameter of a spherical bubble of the same volume as the observed bubble), $D_{e, 0}$ is an estimate of the initial bubble size formed at the distributor (here we have presented their solution appropriate to porous plate distributors), $D_{e, \infty}$ is an estimate of the "maximum bubble size attainable by coalescence," $x$ is the distance above the distributor, $D$ is the equivalent bed diameter, $U$ is the superficial gas velocity and $U_{m r}$ is the minimum fluidization velocity. Notice that this correlation is independent of particle size, density and the depth of the bed. The only problem with using this correlation is that the equivalent bubble diameter soon reaches a state where it exceeds the width of our bed. (Such a condition is generally considered to correspond to the onset of slugging.) Thus we only use this correlation when the bubble diameter is smaller than the dimensions of the bed after which point we assume that the bed is slugging so that the bubble size equals the bed size.

The resulting dimensionless particle pressure $P_{p} / \rho_{p} g D_{e}$ is plotted in Figure 3.4.8 as a function of the void fraction $c$. As the bubble diameter is undefined when the bed is fixed, only the points after minimum fluidization are included in this plot. It appears that, when plotted this way, $P_{p} / \rho_{p} g D_{e}$ is nearly constant and equal to 0.08 . Especially interesting is the fact that the rise in particle pressure that occurs right after minimum nuidization is well accounted for. We therefore conclude that the particle pressure in a gas nuidized bed scales with the bubble diameter. This reflects the fact that, when fully fluidized, the bubbles are driving the internal agitation of the bed, a manifestation of which is the particle pressure exerted on the side wall.

You might notice in Figure 3.4.8, that there is some scatter in the data when the bed is slugging. This is due to the assumption above that the bubble diameter in this regime equals the bed size. This is a common assumption although it is clearly false as the volume of a slug will grow as it moves upward through the bed. The effect of this on the particle pressure is evident in our earlier observation (shown in Figure 3.4.2) that the particle pressure increases slightly going upward through the bed, even when the bed is slugging. Thus, one would expect that, as we don't take this growth into account in the above scaling, the data taken in the slugging region with the probe at the $7.6 \mathrm{~cm}$ location would have slightly lower pressure than the $15.2 \mathrm{~cm}$ location which in turn is lightly lower than the $30.5 \mathrm{~cm}$ location. Exactly this behavior is apparent in the scatter apparent at large void fraction in the above 


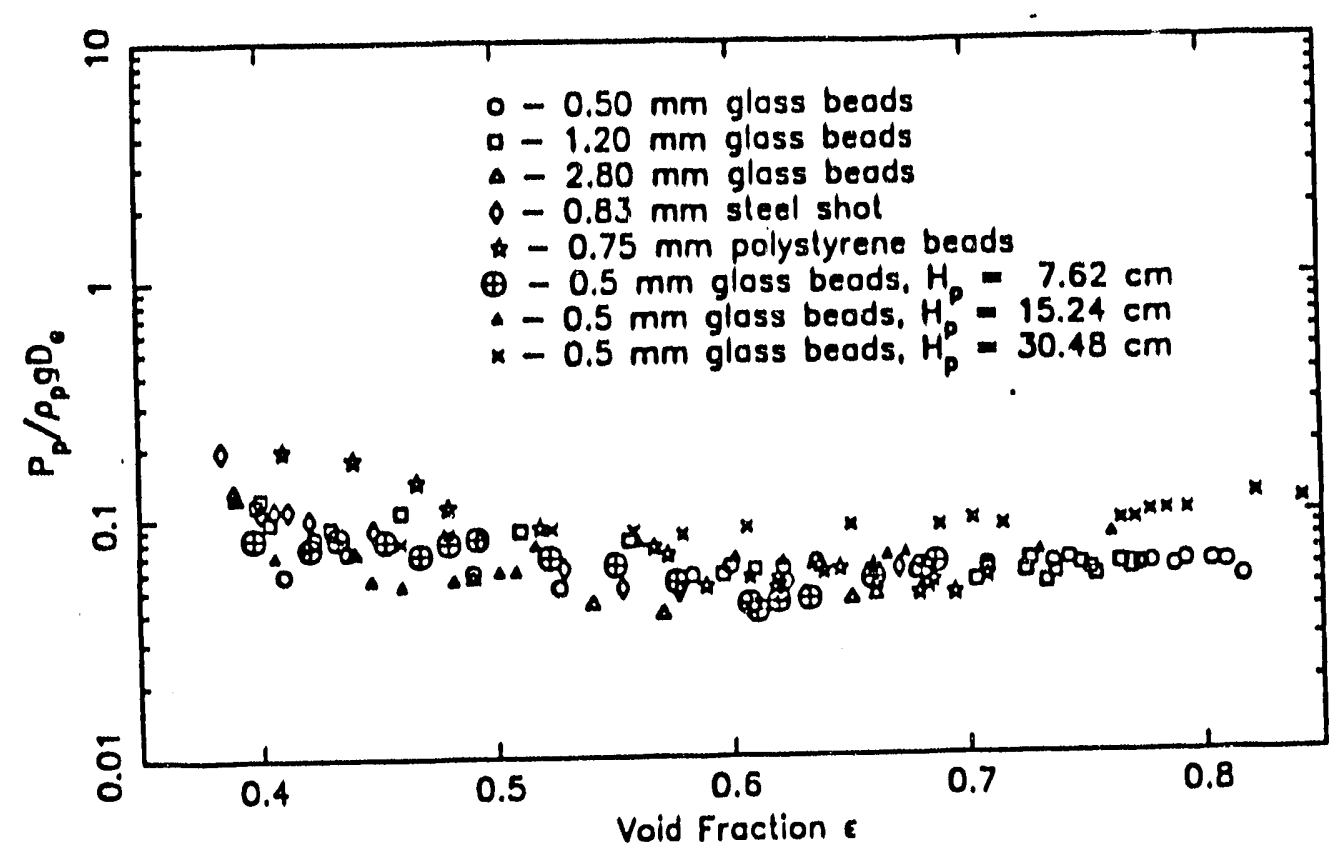

Figure 3.4.8: The particle pressure scaled by the particle density and the effective bubble diameter taken from the correlation of Mori and Wen (1975). Note that, with this scaling, the particle pressure is independent of everything. In particular, the initial rise in the particle is exactly modeled by the bubble growth. Here, $\mathrm{H}_{\mathrm{p}}$ refers to the height of the probe above the distributor.

figure. It does not, however, explain the scatter at smaller densities, which may be simply excused as a rellection of the inaccuracies inherent in the Mori and Wen correlation, or it could reflect physical phenomena, such as the pressure generation due to thermal-like motions of individual particle, that are also not accounted for in the above scaling.

But also notice that the $P_{p} / \rho_{p} g D_{e}$ scaling explains why the particle pressure appears to approach a constant value at the higher velocities when the bed is slugging. Even though the slug size is not equal to the effective diameter of the bed, the bed size does limit the growth of slugs, thus effectively putting a cap on the largest particle pressures that can be realized. Another thing to note is that bubble velocities scale as $\sqrt{g_{e}}$, indicating that the above expression is equivalent to saying that the particle pressures scales with the square of the bubble velocity. Thus, this dependence on bubble sizc reflects both the amount of material moved by a bubble (which should be related to the bubble size) and the velocity of the motion. One must remember, however, that these are all time averaged quantities, so that the above scaling must also account for the frequency of bubble passage. 


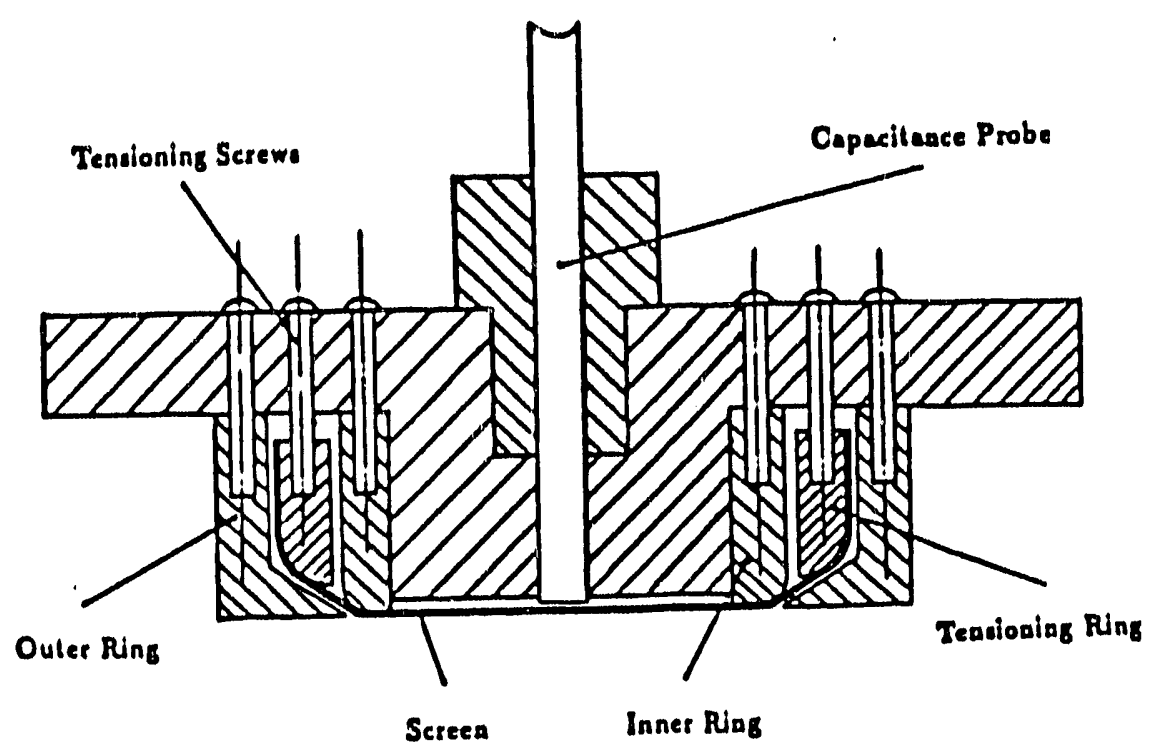

Figure 3.5.1: Schematic of the new particle pressure transducer

\subsection{TIE NEW PARTICLE PRESSURE TRANSDUCER}

Some problems, that should have been apparent in the discussion surrounding the design and testing of the particle pressure transducer in section 3.2. The RC circuit equivalent referred to there is a result of (1) the narrow passages leading to the rear of the probe (large $R$ ) and (2) the alrge cavity behind the diaphragm (large $C$ ). As a result, there was a time delay in the response of the probe that led doubt to any instantaneous particle pressure measurements that might be dervied from it. This led to the design of a new probe which is shown schematically in Figure 3.5.1. Instead of using a solid diaphragm, the design uses a porous screen which is tensioned, much like a drumhead, over the probe. Instead of passing through small boles surrounding the diaphragm, the gas pressure is equalized by simply leaking through the screen. The major advantage of this design is that the large diaphragm area applies a much smaller resistance to the passage of gas across the diaphragm, thus reducing concerns about the response time of the probe to an $₹$ polied particle pressure. This allows us to measure the instantaneous particle pressures.

This allows us to plot time traces of the particle pressure, three sxamples of which are shown in Figure 3.5.2abc. The three cases were chosen to represent the pressure distribution just after minimum nuidization when the bed is just barely bubbling (Figure 3.5.2a), when the bed is freely bubbling (Figure 3.5.2b) and when the bed is slugging (Figure 3.5.2c). All are for $0.5 \mathrm{~mm}$ glassbeads with the probe mounted at the $15.2 \mathrm{~cm}$ location within a bed that was initiaily $31.8 \mathrm{~cm}$ deep. (This corresponds to the square points plotted in Figure 3.4.2.) Just past minimum nuidization, the particle pressure is 


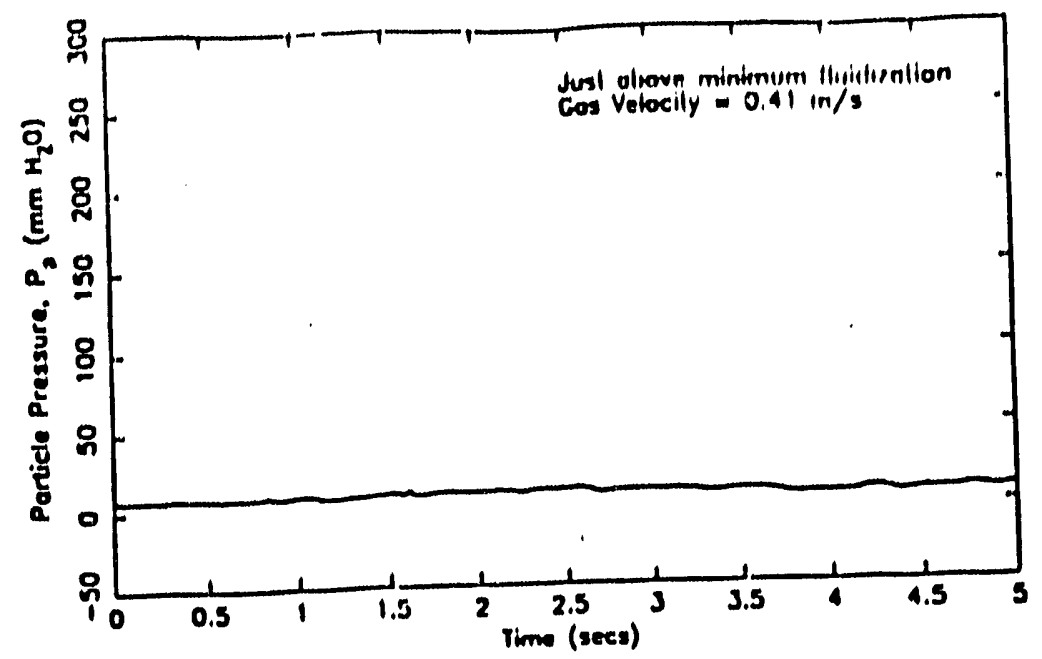

(a)

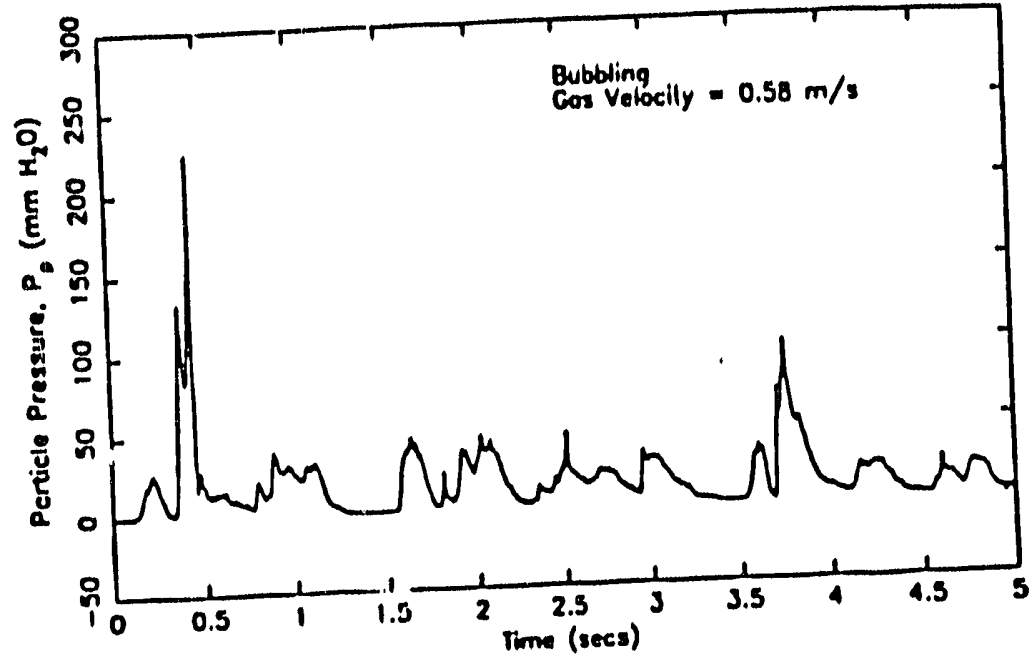

(b)

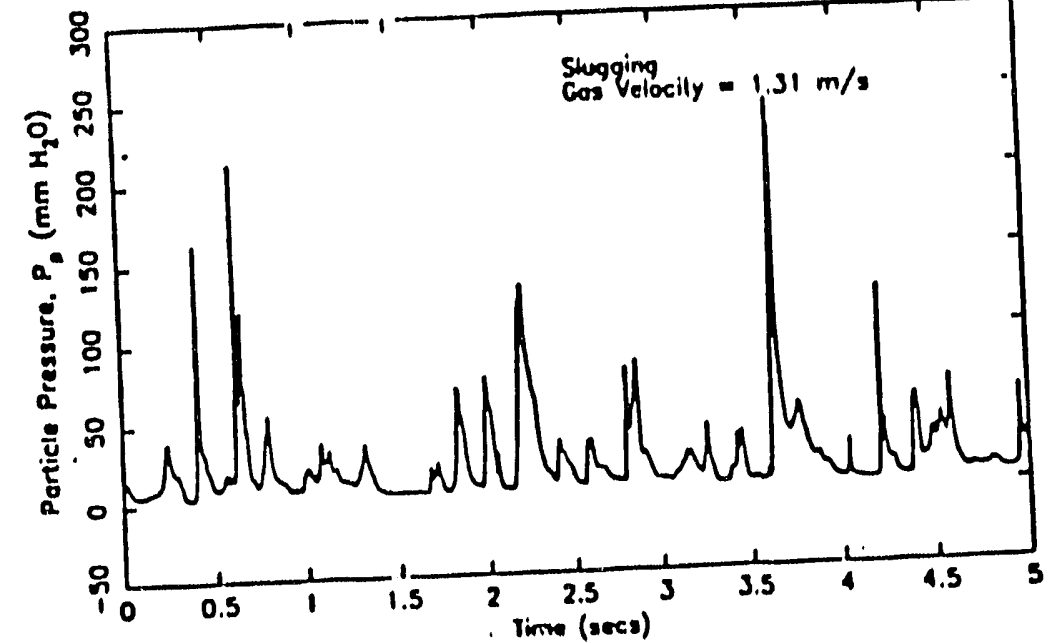

(c)

Figure 3.5.2: Time traces of the particle pressure, measured using the new probe. (a) just beyond minimum nuidization, (b) when the bed is freely bubbling and (c) when the bed is slugging. All of the dala is for $0.5 \mathrm{~mm}$ glassbeads. 
small but nearly constant, reflecting the pressure applied by the thermalized motion of the particles. Bubbles and slugs, however, generate large scale fluctuations, as seen by the peaks in the curves. By comparing with Figure 3.4.2, one can see that the fluctuations are many times the average particle pressure. Notice that the magnitudes of the peaks when the $t$ : $d$ is slugging are not greater than the largest peaks when the bed is simply bubbling, although the large peaks occur more frequently in the slugging bed. This information should be useful in determining the maximum forces to which particles are subject and thus provide insight into the attrition of particles within a fluidized bed. We also hope to, eventually, examine the particle pressure that are generated by the passage of individual bubbles, similar to the fuid pressure measurements made by Littman and Homolka (1973).

However, no attempt has yet been made to determine just how the bubbles apply the particle pressure. Part of the problem is that it is impossible to determine the location of bubbles in the bed relative to the position of the probe and thus one cannot determine how the particle pressure is related to the position of the bubble. However, this is not as large a problem when the bed is slugging as the slugs fill the bed and their location is easily visible through the sidewalls.

Figure 3.5.3 shows the time trace of the particle pressure due to the passage of a single slug. Here, the probe has been placed just above the original packed bed. Two peaks are apparent. The first and largest occurs as the nose of the slug passes the transducer. Exactly this behavior is predicted by Stewart \& Davidson (1967), the only theoretical prediction of particle pressure of which I am aware. They predict a large generation of particle pressure as particles are forced against the wall as they pass around the nose of the slug. (However, the magnitudes of the predicted particle pressures are much smaller than those observed here.) This is an indication that the particle pressure is not uniformly distributed across the bed. However, only a small fraction of the particle will actually pass around the nose of the slug. Most will be lifted by the slug, only to crash back as the slug disintegrates. This crashback accounts for the second peak in the trace.

\subsection{PARTICLE PRESSURES IN GAS-FLUIDIZED BEDS UNDERGoING PARTICULATE FLUIDIZATION}

Our previous studies indicated that the particle pressures generated in a gas-fluidized bed were primarily a byproduct of the motion of bubbles in the bed. However, the materials used in that study fell into types B and D on the Geldart classification and, consequently, the beds bubbled immediately after minimum fluidization. Hlowever, small, lightweight material undergo an intermediate stage of "particulate nuidization", in which the pressure drop across the bed is large enough to support the 

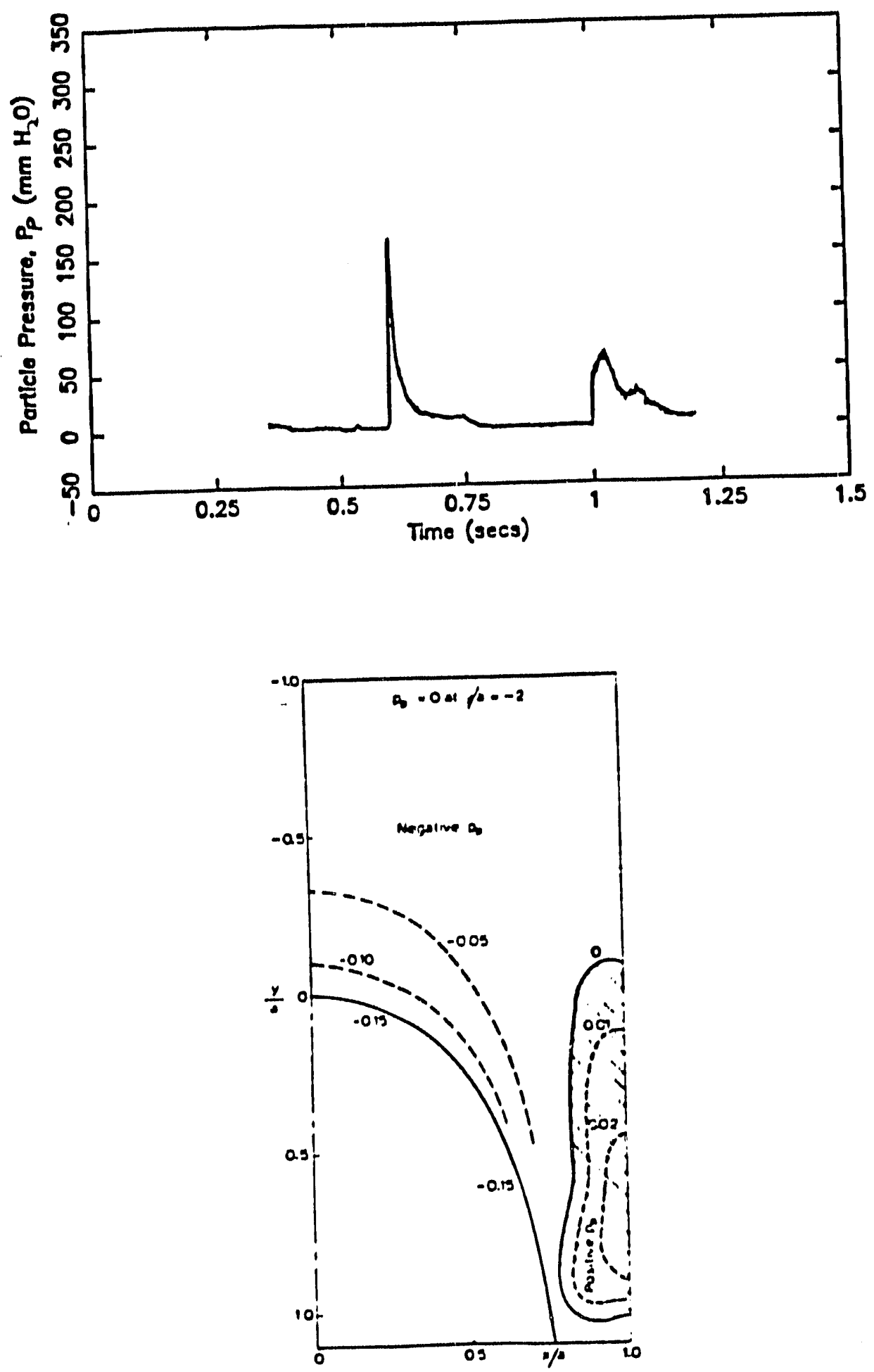

Figurc 3.5.3: Top: a time trace of the particle pressure signal as a slug passes the probe. The first peak represents the passage of the nose of the slug and the second, the crash back of the material lifted by the slug Bottom: The predictions of Stewart and Davidson, showing a region of large particle pressure generated along the wall by the nose of the slug. 


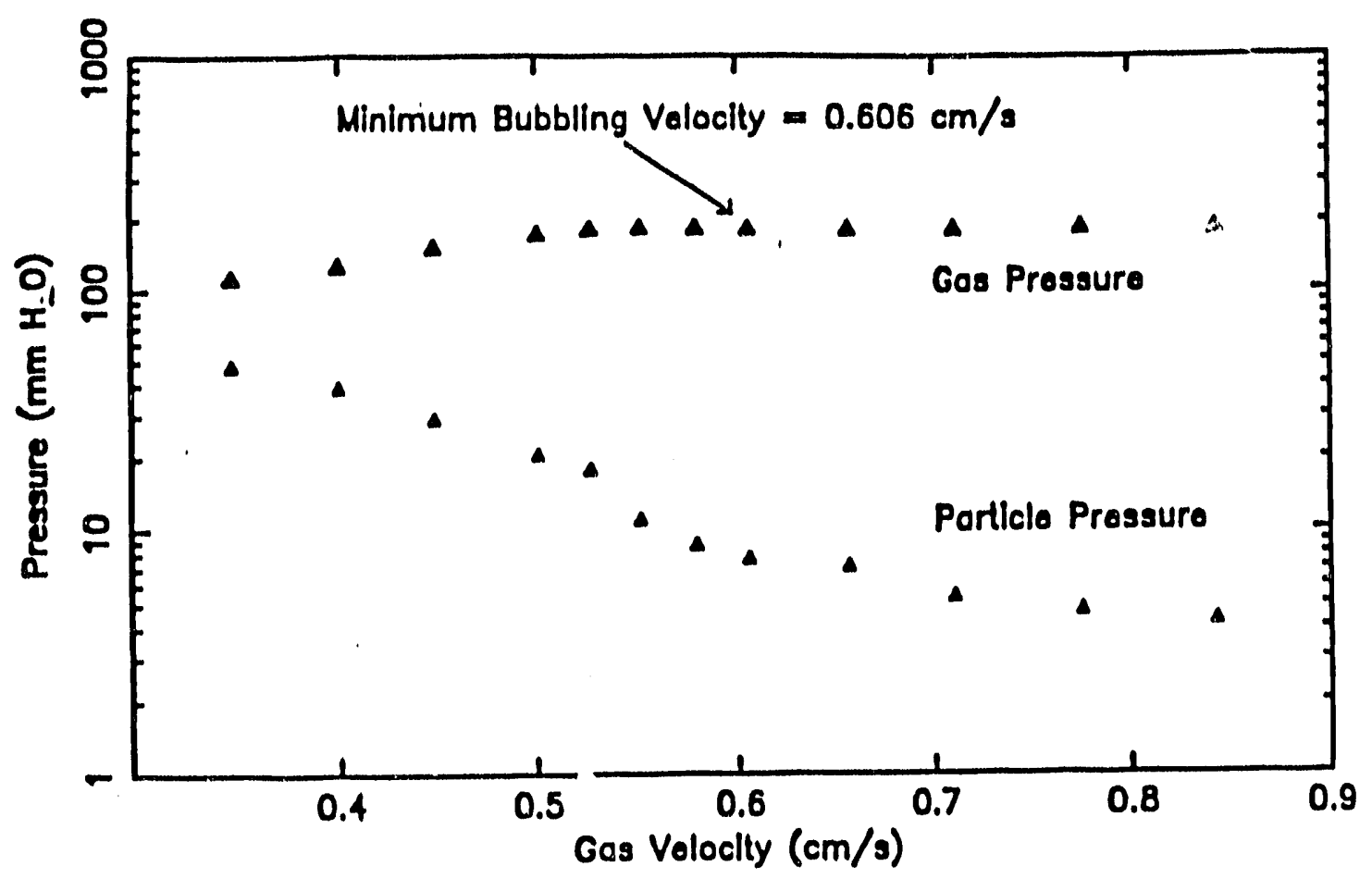

Figure 3.6.1: Measurements of the particle pressure in a homogeneously nuidized bed of cracking catalyst.

weight of the bed, but no bubbles appear. All early stability studies of nuidized beds predicted that the bed should be unstable under all circumstances and much of nuidization modeling has been concerned with explaining the stability of the bed in this region.

Early on, it was shown that the particle pressure could be a stabilizing mechanism. Essentially, the particle pressure introduces a compressibility into the solid phase of the bed. The compressibility resists the voidage instabilities that grow into bubbles and stabilize the beds. On a microscopic level, it was speculated that the particle pressure reflected the random, almost thermal, nuctuation of individual particles. The velocity nuctuations, in turn, are generated by the randomness of the gas now to which a particle is immediately exposed. Locally, the direction of the gas now that a particle experiences is determined by the tortuous path the gas follows as it works its way upward through the bed and may change with time as neighboring particles move around.

However, in the past few years, there bave been indications that a completely different mechanism is at work. Such small particles are naturally cohesive. Consequently, a bed could remain stable as long as the disturbances are not large enough to break down the interparticle bonds. The 
earliest indications that this might be the case were presented by Mutsers \& Rictema (1977). These describe experiments in which a bed of homogeneously fluidized material was tilted with respect to the vertical. If the bed were truly nuidlike, the free surface of the bed would move to remain horizontal as the angle was changed. But the observations showed that the the free surface tilted with the bed and could maintain a substantial angle with respect to the horizontal. This indicated that the bed material could withstand a yield stress. They speculated that the source of this yield stress was the unbroken contacts between particles. Recently this idea has received support from some experiments by Jackson (personal communicatrion) in which he made extremely detailed density measurements across a particulate fluidized bed. These showed significant vold fraction variations within an apparently homogeneous bed that were stable over time scales on the order of hours. Interestingly, the void fractions variations were nearly sinusoidal in shape as if a void fraction wave was frozen in place as it passed upwards through the bed. Jackson speculated that such structures could only be maintained if there were strong interparticle forces.

Obviously, some insight into this phenomenon could be had by examining the particle pressures generated in the homogeneous regime. This is easier than it sounds, largely because materials may only be homogeneously nuidized with great care. Of particular concern is the design of the distributor as any inhomogeneities there will grow into bubbles. After much trial and error, we discovered that a distributor could be built from multiple sheets (ten is a good working number) of chemical filter paper. The results of test performed on FCC are shown in Figure 3.6.1. This shows the gas pressure drop across the entire bed and the particle pressure. Here the particle pressure probe was placed 3 inches above the distributor in an FCC bed that was initially $8 \frac{1}{2}$ inches deep. The experiments were started with the bed bubbling lightly and the points show what haprens as the gas velocity is reduced. Bubbles disappeared from the free surface at a superficial gas velocity of $0.606 \mathrm{~cm} / \mathrm{s}$ (although it is likely that no bubbles are seen at significantly higher velocity at the depth where the probe it placed.)

The results show that the particle pressure is a continuously decreasing function of the fluidizing gas velocity even throughout the region of particulate fluidization. This is incompatible with the idea that particle pressure in that regime is generated by the thermalized motion of particles. Under those conditions, one would expect that the larger the gas velocity, the larger the disturbances that a particle experiences and the larger the particle pressure that would be generated. Consequently, if such a mechanism were at work, one would expect that the particle pressures would rise as the superficial gas velocity is increased. This indicates that some other mechanism, perhaps strong interparticle forces are the stabilizing mechanism. Furthermore, notice that the particle pressures do not fall to zero, even when the bed is fully supported. This indicates that the there is still some structure, most likely the yield stresses between the particles that can support a particle stress. Consequently, these observations 
support the notion that such a bed is not truly nuidized and, instead, retains many of its solld characteristics.

\subsection{TIE RELATIONSUIP BETWEEN TUE PARTICLE PRESSURE AND IEAT TRANSFER}

This investigation was originally intended to investigate a linkage between the particle pressure and the heat transfer behavior in a nuidized bed. The original argument went something like this: It has long been known that the heat transfer coefficient increases dramatically directly following minimum fluidization. However, further increasing the gas flow, cause the heat transfer coefficient to reach a maximum and, eventually fall slightly. Conventional wisclom accounts for this behavior as a tradeoff between the agitation of the bed and the local particle concentration. When the bed first becomes fluidized, it is highly agitated, primarily due to the onset of bubbling. However, with further increase in the gas velocity, the bubbles grow until they begin to insulate the heat transfer surface causing a reduction in the heat transfer rate. (This is exactly the same argument used to explain the change from nucleate to transition boiling in the boiling curve for normal liquids.) Note, that this is a mechanically based argument. When one combines density with agitation it begins to sound like a pressure and, thus, it was hoped that the heat transfor behavior could be directly related to the particle pressure. This idea was further encouraged by some early measurements of the particle pressure that showed just the kind of maximum seen in the heat transfer curves. However, when we eliminated a potential source of error in the particle pressure probe, that maximum simply went away (we believe that the maximum was induced by airflow behind the diaphragm). It was then apparent from the data that the heat transfer behavior would not mirror the particle pressure.

Still, as it was the main subject of the grant, we felt obligated to measure the heat transfer coefficient and particle pressure simultaneously and thus make a final judgment. The results are shown in Figure 3.7.1 and 3.7.2 for the particle pressure and heat transfer respectively. Both were measured in the side wall of our $5 \times 5$ inch gas-nuidized bed. One can clearly see that there is no maximum in the particle pressure as there is in the heat transfer curve and thus the original hypothesis that led to this phase of the grant must be considered to be false. However, notice that both curves do undergo an initial rise initially after minimum nuidization and both asymptote to a constant at high fowrates. (This is the region in which the bed is slugging heavily.) Now, we have shown that the particle pressure can be related to the bubble size. Hence, the particle pressure goes to a constant when the bed is slugging and the growth of bubbles is limited by the dimensions of the bed. Furthermore, the instantaneous heat transfer behavior has long been related to the passage of bubbles. (Bernis et al. 




Figure 3.7.1: The particle pressure on the sidewall of a gas-nllidized bed of $0.5 \mathrm{~mm}$ glassbeads.



Figure 3.7.2: The corresponding heat transfer cocmicient measured on the sidewall of the same bed. 
(1977) Thus, it is possible that similar processes are imposing a limit on the heat transfer. (I must confess that I have never seen any published results that show the heat transfer coefficient approaching a constant at large flowsates. But then again, probably very little work has been done in this regime as (1) it is far from the optimum heat transfer behavior and (2) I cannot understand why anyone would want to operate a slugging fluidized bed for any other than academic reasons.) It is therefore possible that, in this region, there may be some relationship between the two quantities. Still, we feel that the major value in this work comes from the insights that the particle pressure ineasurements give to the hydrodynamics of fluidized beds and have abandoned the theat transfer work.

\subsection{CONCLUSIONS}

This paper has described measurements of the particle pressure exerted on the side wall of a gas fluidized bed. All the results show the same characteristic behavior: At zero superficial gas velocity, the particle pressure is large as all of the pressure is supported across interparticle contacts. Turning on the gas flow reduces the particle pressure on the walls as progressively more of the material is supported by fluid forces. The particle pressure reaches a minimum at the point where the bed is fully supported, and, with a further increase in gas velocity, bubbles appear and the particle pressure begins to rise again due to the agitation of the bed by bubbles.

When the bed is fully nuidized, the particle pressure appears to be dominated by the bubble motion and the particle density. A series of tests revealed that, in the fully fluidized region, the particle pressure varies as:

$$
\frac{\mathrm{P}_{\mathrm{p}}}{\rho_{\mathrm{p}} \mathrm{g} \mathrm{D}_{\mathrm{e}}}=\text { constant } \simeq 0.08
$$

where $\rho_{\mathrm{p}}$ is the particle density, $\mathrm{g}$ is the gravitational acceleration, and $\mathrm{D}_{\mathrm{e}}$ is the effective bubble diameter. $D_{e}$ was determined in a rather ad hoc manner using the correlation of Mori and Wen (1975) until the bubble diameter equaled the effective diameter of the fluidized bed, after which it was assumed to be constant and equal to the average equivalent bed diameter. But, despite the obvious deficiencies of the model, all of the data collapsed, nearly to a point, when plotted as above. In particular, the initial growth in the particle pressure after minimum nuidization correlates with the change in bubble size.

The passage of a bubble causes large scale agitation of the particles. In other words, as the bubtle 
size is very much greater than a particie diameter, it causes the movement of many particle masses. This is a very different picture from the force transmission by individual impacts seen by Kumar et al. (1990) as the mechanism leading to the particle pressure in liquid nuidized beds. Similar behavior may occur in the particulate regime of gas-fluidized beds before bubbles appear. However, to see such events would require performing tests on very small (less than about $100 \mu \mathrm{m}$ diameter) test materials for which this type of probe may not be suitable as the lubrication forces between the particle and the flat wall surface may significantly slow the particle before impact. (Exactly this behavior was encountered for small, light, particles by Kumar et al. (1990).) While this may give an accurate account of the particle forces on the wall, the measurements will no longer reflect the behavior within the material. We are currently developing a probe that will overcome these difficulties. (Note that lubrication forces will probably not significantly affect the measurements in the bubbling regime. There the bubbles push blocks consisting of many particles against the diaphragm, yet the lubrication forces will be due to only those particles that actually contact the diaphragm. Consequently, one can count on the inertia of a multiparticle block to to overcome the lubrication effects generated at the diaphragm by a such small fraction of its constituent particles.)

Finally, we have developed a new particle pressure transducer that is capable of determining instantaneous particle pressures. The results show that the particle pressures are applied in sharp peaks, with a magnitude of many times the mean particle pressure, due to the passage of bubbles. One interesting observation is that, at least in the slugging regime, the largest peak is applied by the passage of the nose of the slug. Furthermore, a cursory examination of the behavior of a homogeneously nuidized bed has been carried out that indicates that the particle pressure does not vanish during this phase. This indicates that some structure still exists that can support interparticle forces - an idea that is consistent with many recent observations of fuidization in this regime. 


\subsection{APPENDIX - EXPERIMENTAL INNOVATIONS}

Almost every experimental project faces difficulties that have to be overcome. Sometimes these requires minor innovations that are worthy of note because they may aid other researchers. Both of the ideas discussed below, came out of the heat transfer project described in section 2.

\subsection{HEATER CONTROL CIRCUIT}

Problems arose during the shear cell experiments, because, in order to ensure that the heat llux into the mixture is measured accurately, many guard heaters are needed to block all heat paths from the primary heater, other than that going directly into the test material. After many iterations, we found that six heaters configured as in Figure 2.1.2 are required.

Proper operation requires that the temperatures of all the heaters be balanced. It is difficult enough and extremely tedious to balance two heaters manually, but six heaters took a graduate student over a day to stabilize long enough to demonstrate that this configuration would work. The difficulties arise because, changing the heater voltage does not immediately change the heater temperature. Instead, it may take minutes for the heater to stabilize. We made the decision to control the temperature of the heaters by computer. It is somewhat ironic to use a high speed computer to perform such a low speed task, but it permits more elaborate heater control schemes to be used than could be performed by a human operator. The schemes we are testing at the moment updates the heater voltages every 5 seconds based on a prediction of what the temperature will be 30 seconds in the future. Consequently, we expect better heater control than could be obtained manually.

The heaters are controlled by six D to A convertors, two of which reside on a Scientific Solutions Labmaster board and four on a Scientific Solutions DADIO board, both mounted in an clone of an IBM AT computer. Control circuitry, illustrated in Figure 4.1.1, is required to interface the convertors to the heaters. The circuit is used to allow the $0-5$ volt, low current output of the $D$ to $A$ converters to be used to control the $0-40$ volt high current required by the heater. The circuit is simple but, as mechanical engineers, it took us a long time to come up with it. (Many thanks are due here to my neighbor Carver Mead.) Basically, it is a simple coupled ernitter amplifier created from a Darlington transistor. However, such a circuit is thermally unstable. To eliminate the temperature instability a 741 op-amp is added. The op-amp balances the input voltage to the collector voltage of the transistor (which is reduced from a $0-40$ volts to a $0-5$ volt range by a voltage divider composed of $R 3$ and $R 4$ ). This setup allows very accurate setting of the voltage through the transistor. Note, however, that the collector voltage is large when the heater current is small. Hence, a 5-volt input corresponds to zero 


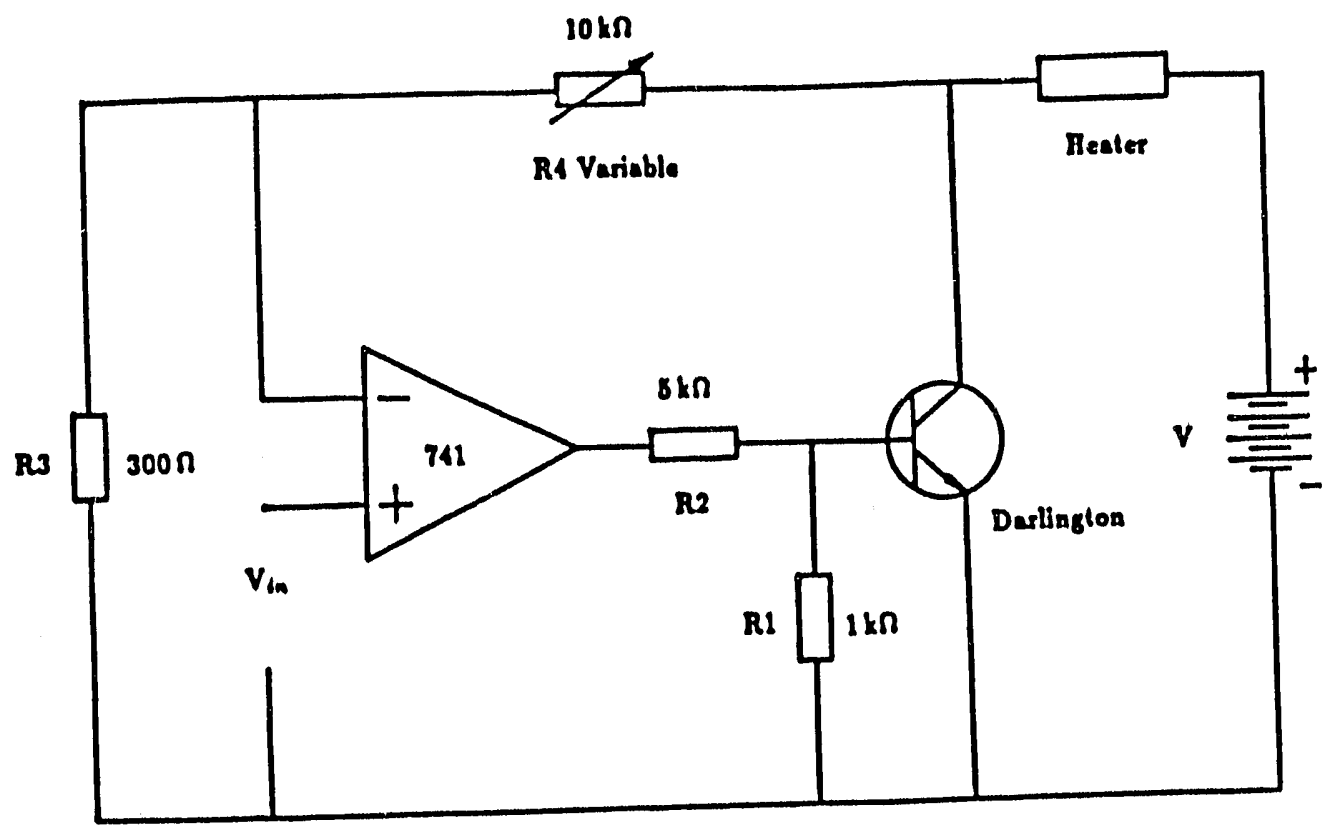

Figure 1.1.1: The heater control circuit

current now and a zero volt input corresponds to maximum current now.

\subsection{TIERMOCOUPLE CONNECTIONS TIROUGI SLIP RINGS}

At the start of this project, the temperatures within the rotating disk were monitored by thermocouples through a slipring assembly and referenced to the temperature, $T_{B}$ of the constant temperature bath that is used to cool the cold side of the shear cell. This configuration is shown in Figure 4.2.1a. We began to notice slight inconsistencies in the measurements made from day to day and were finally able to relate the problem to the sliprings. Like thermocouples, the connections made between the thermocouple wires and the sliprings mate dissimilar materials and, consequently, act somewhat as thermocouples themselves. As the sliprings were heated by friction or by changes in the ambient temperature of the room, they would slightly throw of the calibration of the thermocouples. We came up with a solution to this problem by using a new thermocouple circuit which is shown in Figure 4.2.1b. Please note in reading this figure that we are using Type $\mathrm{T}$ or Copper (Cu) Constantan (C) thermocouples. Consider thermocouple 1 as monitoring the temperature of interest. The initial problem arose because the connections between the $\mathrm{Cu}$ and $\mathrm{C}$ legs and the sliprings would creatc different junctions and thus generate different voltages at different slipring temperatures. This problem is solved by inserting into the $C$ leg, thermocouple 2 which is reverse junctioned so that its $C$ leg is connected to the $\mathrm{C}$ leg of thermocouple 1. The junction of thermocouple 2 senses the ambient air in the 
(1)

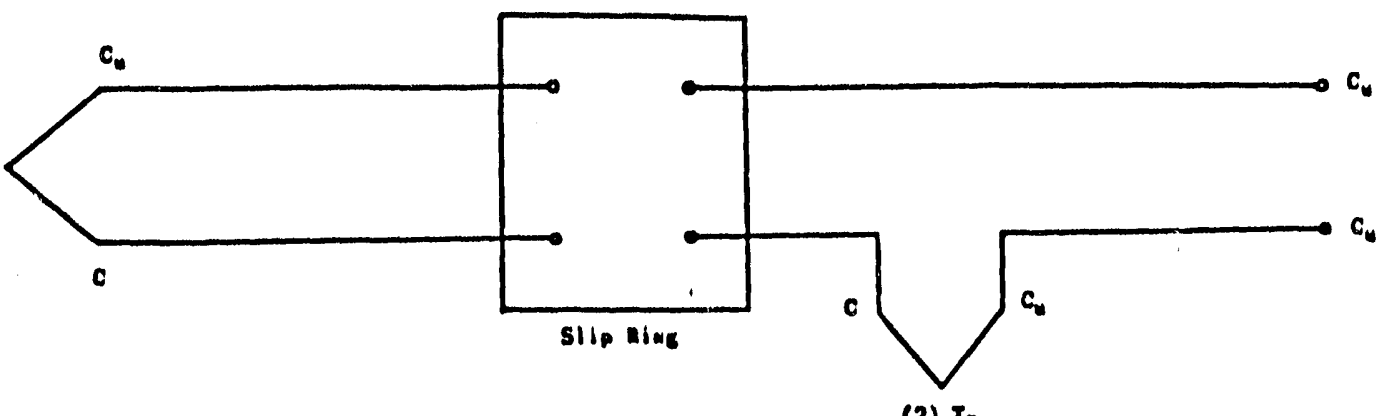

(a)



(b)

Figure 6: (a) Diagram of the old thermocouple circuit. (b) new thermocouple circuit

room. This acts as a reference thermocouple which references the temperature of thermocouple 1 to the ambient temperature in the room. It has the added advantage that the connection with the sliprings is now made through the $\mathrm{Cu}$ leg of thermocouple 2, which generates the same voltage as the connection of the $\mathrm{Cu}$ leg of thermocouple 1. Thus, the two voltages cancel each other and the slipring assembly no longer takes part in the temperature measurement. The disadvaritage is that the temperature measured by thermocouple 1 is now referenced to the ambient air which may change during the course of the experiment. This problem is eliminated by the insertion of two additional thermocouples, 3 and 4 . Thermucouple 3 is reversed junctioned to thermocouple 2 and, like thermocouple 2, senses the temperature of the ambient air and is held as close as possible to a point of passage of the rotating thermocouple 2 - but on the stationary side of the slipring. Thus thermocouple 3 cancels any voltage generated at thermocouple 2. Finally, thermocouple 4, which is immersed in the constant temperature bath which cools the cold side of the shear cell, is added to the system reverse connected with thermocouple 3 - and consequently, is reversed junctioned to thermocouple 1 - to provide the global 
reference temperature for the measurement. Note that the output leg of thermocouple 1 is the $\mathrm{Cu}$ junction so that no further downstream connections - as long as they are made of the same materials will create additional junctions. In short, thermocouples 2 and 3 are passive players in this circuit and are used only so that the slipring assembly does not play a role in the temperature measurement. (It helps if thermocouples 2 and 3 are either slightly insulated, or connected to large thermal capacitances in order that their temperatures not change too quickly in response to minor changes in room temperature such as when the heating goes on. Otherwise, as one thermocouple rotates with the shear cell, it will reflect the temperature change faster than its stationary counterpart, producing a false reading. Insulation or large thermal capacitance slows the response of the thermocouples to fast changes in room temperature.) Globally, the output reflects the temperature of thermocouple 1 referenced to the constant temperature bath through thermocouple 4. 


\section{REFERENCES}

Bagnold, R.A. 1954 Experiments on a gravity-free dispersion of large solid spheres in a Newtonian Ruid under shear. Proc. Roy. Soc. London, Ser., A225, 49.

Batchelor, G.K., 1988, A new theory for the instability of a uniform nuidized bed, Journal of Fluid Mechanics, $19375-110$

Bernis, A., Vergnes, F. and Le Gofr, P., 1977, Influence du passage d'unc bulle sur le coefficient instantané de trasfert de chaleur à une paroi immergée dans un lit fluidisé, Powder Technology, 18 267-276

Campbell, C.S., 1987, A technique for determining the nuidization mechanism in horizontal slurry flow, Proceeding of the Solids Transport Contractor's Review Meeting, September 17-18, 1987, Pittsburgh, Pennsylvania, 68-77, US Department of Energy, Pittsburgh Energy Technology Center

Campbell, C.S. 1082, Shear flows of granular materials. PhD Thesis and Rep. E-200.7, California Institute of Technology., Pasadena, Ca.

Campbell, C.S., 1986, Computer simulation of rapid granular flows, Proc. $10^{\text {th }}$ US National Congress of Applied Mechanics, Austin Texas, June 1986, ASME, New York, 327-38

Campbell, C.S. 1989 The stress tensor for simple shear flows of a granular material. J. Fluid Mech., 203, 449.

Campbell, C.S. 1990, Rapid granular flows, Annual Review of Fluid Mechanics, 22, 57-92

Campbell, C.S. \& Brennen, C.E. 1985, Computer simulation of granular shear flows. J. Fluid Mech., $151,167$.

Campbell, C.S. \& Gong, A. 1986, The stress tensor in a two-dimensional granular shear flow, J. Fluid Mech, 161, 107-125

Campbell, C.S. \& Gong, A., 1987, Boundary conditions for two-dimensional granular flows, Proceedings Sino-US International Symposium on Multiphase Flows, Hangzhou, China, August, 1087, Volume I, 278-283, Zhejiang University Press, Hangzhou, China

Campbell, C.S. \& Wang, G.D. 1986 The apparent conductivity of shearing particle flows. Proc. Int. $8^{\text {th }}$ Heat Transfer Conf., 4, 2567-72. (Washington, DC: Hemisphere).

Campbell, C.S. and Wang, D.G., 1990, A particle pressure transducer, suitable for use in gas-fluidized beds, Measurement Science and Technology, accepted

Chung, Y.C. \& Leal, L.G. 1982 An experimental study of the apparent thermal conductivity of a sheared suspension of rigid spheres. Int. J. Multiphase Flows., 8, 605.

Craig, K., Buckholtz, R.II. \& Domoto, G., 1987, The effects of shear surface boundaries on stresses for the rapid shear of dry powders, Trans. ASME J. Tribology, 109, 232-237

Hanes, D.M. 1983 Studies on the mechanics of rapidly flowing granular-fluid materials. PhD Thesis, Univ. Calif., San Diego, La Jolla.

Hane's, D.M. \& Inman, D.L. 1985 Observations of rapidly nowing granular-nuid materials. J. Fluid Mech., 150, 357. 
Hunt, M.L. and Hsiau, S.S., 1190, Thermal conductivity of granular nows, Proc. Int. $9^{\text {th }}$ Heat Transfer Conf., 177-182 (Washington, DC: Hemisphere).

Jackson, R., 1985, Hydrodynamic stability of nuid-particle systems, in Fluidization, (J.F. Davidson, R. Clift, and D. Harrision eds.) Academic Press, London, 47-72

Jones, A.L. and Wheelock, T.D., 1970, The electrical resistivity of nuidized carbon particles: significant parameters, Chemical Engineering Progress Symposium Series 66, No. 105, 157-166

Kumar, S., Hart, D.P. and Brennen, C.E., 1990, Granular pressure measurements in nuidized beds, ASME Cavitation and Multiphase Flow Forum, June 1990, Toronto Canada

Leal, L.G. 1973 On the apparent conductivity of a dilute suspension of spherical drops in the limit of low particle Peclet number. Chem. Eng. Comm., 1, 21.

Lin, C.J., Peery, J.H. \& Schowalter, W.R. 1970 Simple shear flow around a rigid sphere; Inertial effects and suspension rheology. J. Fluid Mech., $4,1$.

Littman, H. and Homolka, G.A.J., 1973, The pressure field around a two-dimensional gas bubble in a fluidized bed, Chemical Engineering Science, 28, 2231-2243

Lößelmann, G., 1989, Theoretische und experimentelle Untersuchungen zur Schüttgut-WandWechselwirkung und zum Mischen un Entmischen von Granulaten, Dr.-Ing Theisis, Universitat Fridericiana Karlsruhe (Technische Hochschule), Karlsruhe, Germany, 217 pp.

Lun, C.K.K. Savage, S.B. Jeffrey, D.J. \& Chepurniy, N. 1984, Kinetic theories for granular flow: inelastic particles in Couette flow and slightly inelastic particles in a general flowfield, J. Fluid Mech. 140,223

Mori, S. and Wen, C.Y., 1975, Estimation of bubble diameters in gaseous fluidized beds, AICHE Journal, 21, 109-115

Mutsers, S.M.P and Rietema, K., 1977, The effect of interparticle forces on the expansion of a homogeneous gas-fluidized bed, Powder Technology, 18 239-248

Murray, J.D., 1965, On the mathematics of fluidization Part 1. Fundemental equations and wave propogation, J. Fluid Mech. 21 465-493

Nir, A. \& Acrivos, A. 1976 The effective thermal conductivity of sheared suspensions. J. Fluid Mech., 78,33

Patton, J.S., Sabersky, R.H., and Brennen, C.E., 1986, Convective heat transfer to rapidly flowing granular rnaterials, Int. J. Heat Mass Trans., 29, 1263-69

Reed, A.K. and Goldberger, W.M., 1966, Electrical behavior in nuidized beds of conducting solids, Chemical Engineering Progress Symposium Series 62 No. 67, 71-75

Reynolds, O., 1874, On the extent and action of the heating surface of steam boilers, Proc. Lit. and Phil. Soc, Manchester, 14, 7-12. See also Papers on Mechanical and Physical Subjects, Vol. 1, 81-85, Cambridge University Press, 1900

Savage, S.B. \& Sayed, M. 1983 Stresses developed by dry cohesionless granular materials in an annular shear cell. J. Fluid Mech., 142, 391. 
Stewart, P.S.B \& Davidson, J.F., (1967) Slug now in nuidised beds, Powder Technology, 1, 61-80

Sun, J. \& Chen, M.M. 1988 A theoretical analysis of heat transfer due to particle impact. Int. J. Heat Mass Transfer, 31, 969.

Walton, O.R. \& Braun, R.L. 1986a, Viscosity and temperature calculations for shearing assemblies of inelastic, frictional disks, J. of Rheology, 30, 949-980

Walton, O.R. \& Braun, R.L. 1986b, Stress calculations for assemblies of inelastic spheres in uniform shear, Acta Mechanica, 63, 73-86

Wang, D.G., 1991, Mechanics and Beat Transfer for Granular Flows, PhD thesis, University of Southern California, Los Angeles, Ca., USA

Wang, D.G., Sadhal, S.S. \& Campbell, C.S., 1989, Particle rotation as a heat transfer mechanism. Int. J. Heat Mass Transfer, 32, 1413.

Xavier, A.M. and Davidson, J.F., 1985, Heat transfer in nuidized beds: convective heat transfer in nuidized beds, in Fluidization, (J.F. Davidson, R. Clift and D. Harrison eds.), Acadernic Press, London 

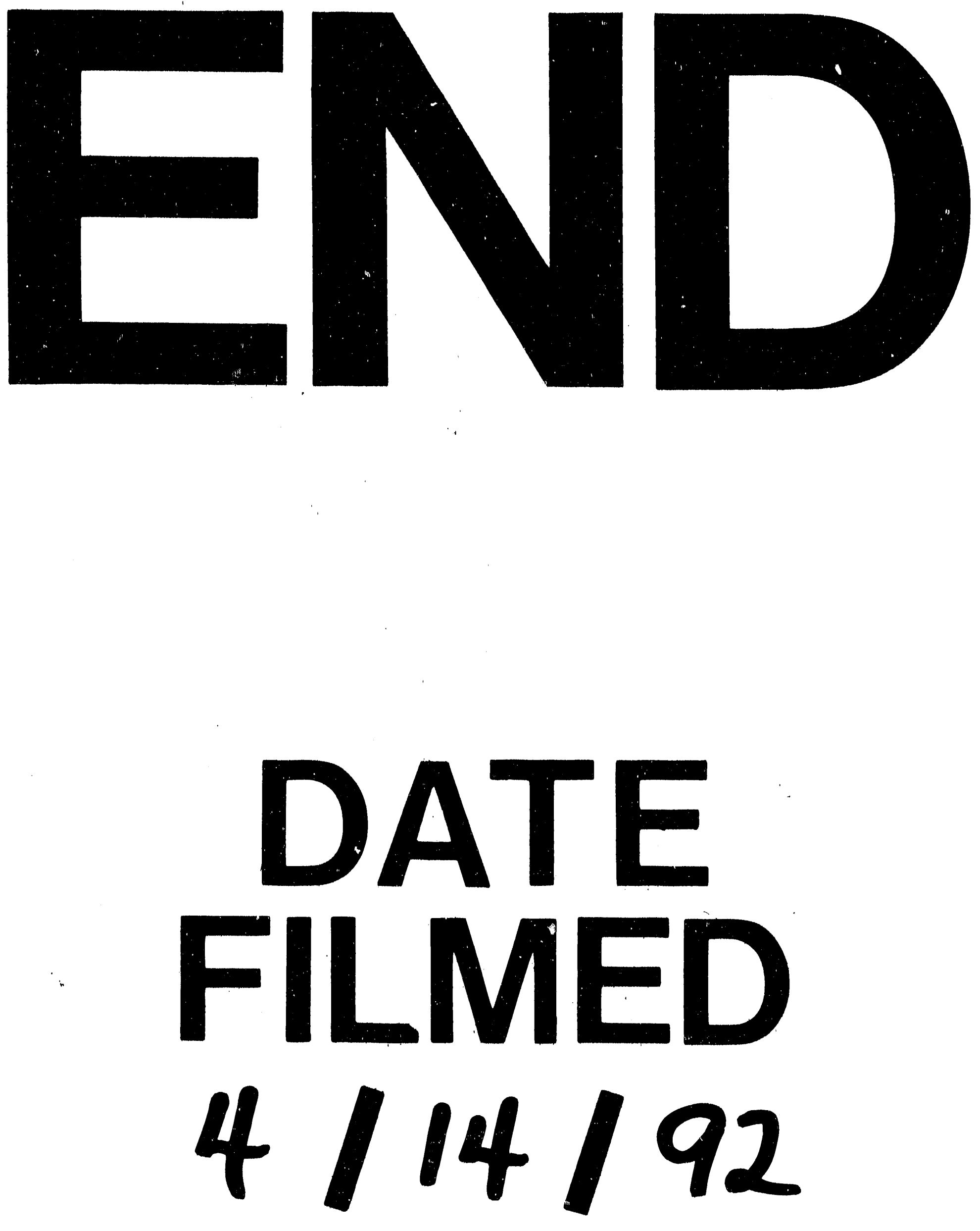


$$
\text { (2) }
$$
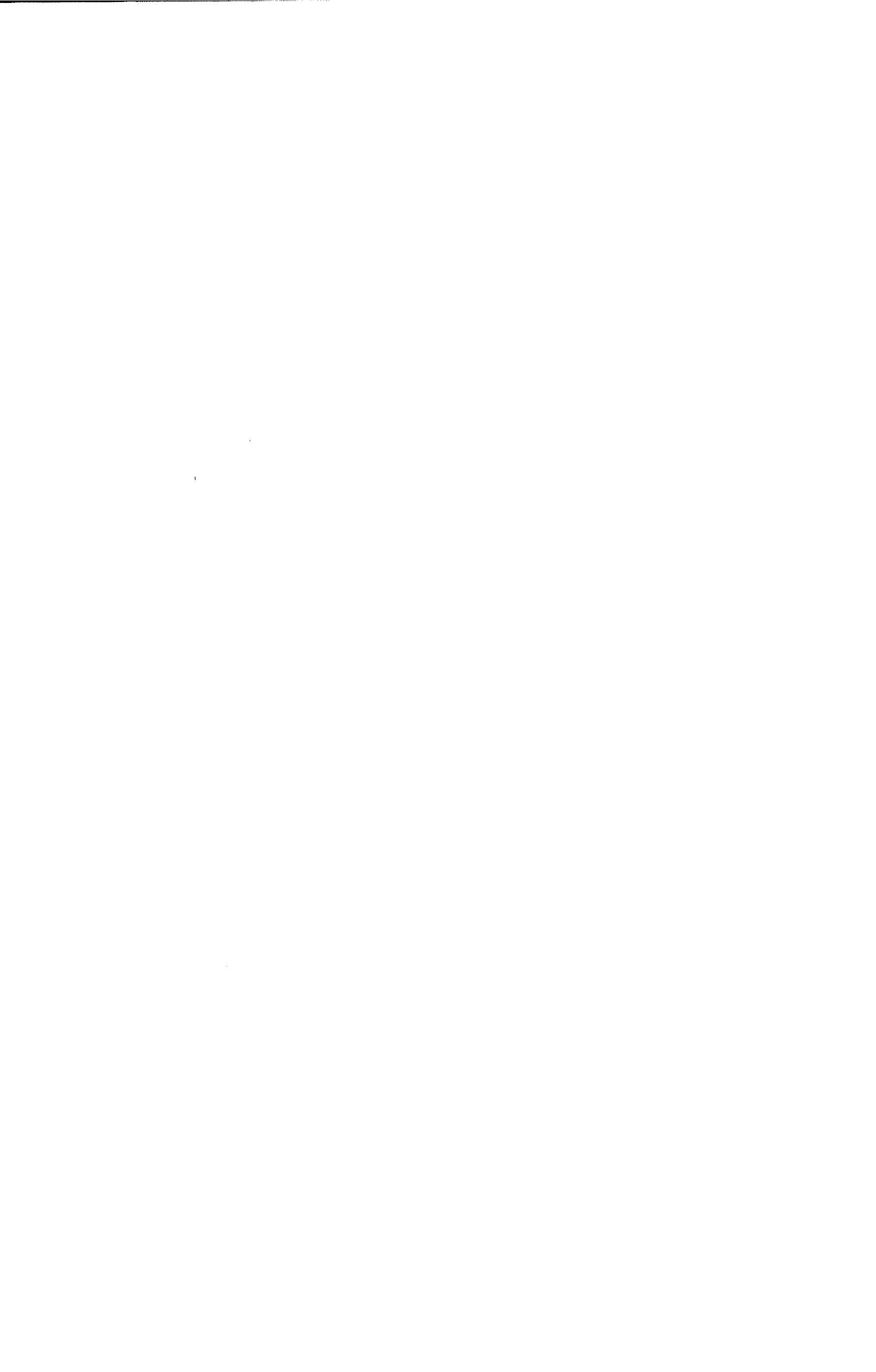